ARTICLE

DOI: $10.1038 / s 41467-017-00080-y$

OPEN

\title{
Yeast Cip1 is activated by environmental stress to inhibit Cdk1-G1 cyclins via Mcm1 and Msn2/4
}

Ya-Lan Chang ${ }^{1}$, Shun-Fu Tseng 1,2, Yu-Ching Huang (1) ', Zih-Jie Shen (10 ', Pang-Hung Hsu (D) ${ }^{3}$, Meng-Hsun Hsieh', Chia-Wei Yang ${ }^{1}$, Silvia Tognetti ${ }^{4}$, Berta Canal ${ }^{4}$, Laia Subirana ${ }^{4}$, Chien-Wei Wang $^{1}$, Hsiao-Tan Chen', Chi-Ying Lin', Francesc Posas ${ }^{4} \&$ Shu-Chun Teng ${ }^{1}$

Upon environmental changes, proliferating cells delay cell cycle to prevent further damage accumulation. Yeast Cip1 is a $\mathrm{Cdk} 1$ and $\mathrm{Cln} 2$-associated protein. However, the function and regulation of Cip1 are still poorly understood. Here we report that Cip1 expression is co-regulated by the cell-cycle-mediated factor $\mathrm{Mcm} 1$ and the stress-mediated factors Msn2/4. Overexpression of Cip1 arrests cell cycle through inhibition of Cdk1-G1 cyclin complexes at G1 stage and the stress-activated protein kinase-dependent Cip1 T65, T69, and T73 phosphorylation may strengthen the Cipland Cdk1-G1 cyclin interaction. Cip1 accumulation mainly targets $\mathrm{Cdk1-Cln3}$ complex to prevent Whi5 phosphorylation and inhibit early $\mathrm{G} 1$ progression. Under osmotic stress, Cip1 expression triggers transient G1 delay which plays a functionally redundant role with another hyperosmolar activated CKI, Sic1. These findings indicate that Cip1 functions similarly to mammalian p21 as a stress-induced CDK inhibitor to decelerate cell cycle through G1 cyclins to cope with environmental stresses.

\footnotetext{
${ }^{1}$ Department of Microbiology, College of Medicine, National Taiwan University, Taipei 10051, Taiwan. ${ }^{2}$ Department and Graduate Institute of Microbiology and Immunology, National Defense Medical Center, Taipei 11490, Taiwan. ${ }^{3}$ Department of Bioscience and Biotechnology, National Taiwan Ocean University, Keelung 20224, Taiwan. ${ }^{4}$ Cell Signaling Research Group, Departament de Ciències Experimentals i de la Salut, Universitat Pompeu Fabra, Barcelona 08003, Spain. Correspondence and requests for materials should be addressed to S.-C.T. (email: shuchunteng@ntu.edu.tw)
} 
$\mathrm{N}$ atural environment changes frequently and wild organisms require proper responses to adapt to the challenge. Under environment-friendly condition, cell cycle proceeds without interruption. However, stress conditions temporarily arrest cells in G1 and further promote proper cellular response to overcome the stress and then re-engage cells into the cell cycle $^{1-4}$. For example, depletion of nutrient ${ }^{5,6}$, osmotic stress $^{7,8}$, and oxidative stress-induced DNA damage all lead to G1 arrest for cells to repair the damage 9 . The G1 checkpoint contains several fine-tuned pathways that monitor cell cycle progression.

In budding yeast, the start transition (START) occurs during late G1 and it is usually considered as a critical initiating point of a new round of cell division ${ }^{10}$. START is a point of no return: post-START cells finish cell cycle irrevocably. Execution of START is mediated by a serine/threonine cyclin-dependent kinase (Cdk1) which physically interacts with one of the G1 cyclins (Cln1, Cln2, or Cln3) to form START-promoting protein kinase complex ${ }^{11}, 12$. Cln3 is the most upstream regulatory G1 cyclin in the transition. The Cdk1-Cln3 complex activates SBF and MBF complexes that drive transcription of START genes $^{13,14}$. SBF associates with its repressor, Whi5, the human $\mathrm{Rb}$ analog, at the target promoters to repress transcription. Cdk1-Cln3 phosphorylates Whi5 to release Whi5 from SBF and activate START transcription ${ }^{15}, 16$. Under osmotic stress, Whi5 and the co-regulator Msal are phosphorylated by the stressactivated protein kinase (SAPK) Hog1 to inhibit G1 cyclin expression for controlling adequate passage through START ${ }^{17}$.

On the other hand, cyclin-dependent kinase inhibitor (CKI) interacts with a Cdk1-cyclin complex to block kinase activity, which plays an inhibitory role. In Saccharomyces cerevisiae, two CKIs were previously well studied. Farl is a pheromone-induced Cdk1-G1 cyclin inhibitor ${ }^{18}$. FAR1 RNA and protein accumulate in early G1 but decline after passing through START ${ }^{19}$. Pheromone treatment triggers the interaction between Farl and Cdk1-Cln to arrest cell cycle for mating to occur ${ }^{20}$. On the other hand, Sic1, an inhibitor of Cdk1-Clb complex, blocks the activities of Cdk1-Clb5/Clb6 and Cdk1-Clb1/Clb2, which are required for DNA replication and mitosis, respectively ${ }^{21}$. Cdk1-Cln1/Cln2-mediated phosphorylation of Sic1 triggers ubiquitin-dependent proteolysis which initiates DNA replication $^{22}$. Sicl prevents cells from premature S-phase initiation, which allows cells to have more time to rescue defects ${ }^{23}$.

$\mathrm{Mcm} 1$ is a MADS family transcription factor ${ }^{24}$ that is highly conserved in all eukaryotes ${ }^{25,}{ }^{26}$. In budding yeast, $\mathrm{Mcm} 1$ is required for pheromone response and also regulates the transcription of multiple cell-cycle genes $^{27-29}$. The $\mathrm{Mcm} 1$ binding site, the early cell cycle box (ECB) element, controls many M/G1 specific genes transcription ${ }^{29}$. Deletion of the ECBs from CLN3 and SWI4 promoters reduces their expression and causes a G1-S delay ${ }^{30}$.

Msn2 and its homologous protein, Msn4, are two key transcription factors that regulate the expression of hundreds of stress response element (STRE)-containing stress response genes $^{31}$. Msn2 and Msn4 are activated to evoke rapid and efficient adaptation responses upon several environmental and metabolic cues, including oxidative stress, heat, osmotic stress, DNA replicative stress, carbon source starvation, and diauxic transition $^{32-35}$. Single deletion of MSN2 or MSN4 causes no visible phenotype, while $m s n 2 m s n 4$ mutants are hypersensitive to general stresses ${ }^{31}$.

A new G1 CKI, Ypl014w (Cip1) has recently been identified in Saccharomyces cerevisiae ${ }^{36}$. Cip1 associates with Cdk1 and $\mathrm{Cln} 2^{36}$. However, the exact regulatory mechanism of Cip1 to control cell cycle progression is still unclear. Here we demonstrate that Cip1 expression is co-regulated by the cell cycle-mediated factor Mcm1 and stress-mediated factors Msn2/4. CIP1 expression fluctuates with cell cycle and peaks at G1. Overexpressed Cip1 binds to all Cdk1-G1 cyclin complexes and blocks cell cycle at G1. At early G1, Mcm1-mediated accumulation of Cip 1 mainly targets to Cdk1-Cln3 complex for preventing the inhibitory phosphorylation of Whi5, inhibiting CLN1/CLN2 expression, and blocking cell cycle progression. Under hyperosmotic stress, Hog1 phosphorylates Cip1 which might increase the association between Cip1 and Cdk1-G1 cyclin complexes. Cip1 plays a functionally redundant role with another CKI, Sic1. These findings suggest that multiple mechanisms regulate Cip1 expression and activity to control the cell cycle.

\section{Results}

Overexpression of Cip1 induces G1 arrest. A previous study demonstrated that Cip1 interacts with the Cdk1-Cln2 complex ${ }^{36}$. To confirm this and further identify the biological function of Cip1, CIP1 was overexpressed under the GAL1 promoter. The budding index analysis showed that overexpression of Cip1 increased unbudded cells from 36 to 55\% (Fig. 1a). As previously reported ${ }^{36}$, Cip1 overexpression caused cell cycle inhibition when cells were released from G1 arrest (Fig. 1b). These data confirm that Cip1 specifically impedes G1 progression when it is highly expressed.

Farl is a pheromone-induced $\mathrm{CKI}^{18-20}$, and Sic1 specifically targets Cdk1-Clb under several stresses ${ }^{21-23}$. To determine whether Cip1-induced G1 arrest was Far1- or Sic1-dependent, FAR1 or SIC1 was deleted in cells overexpressing Cip1. Cip1 overexpression in wild-type, farl, sicl, and farlsicl strains exhibited no difference on growth retardation (Fig. 1c), suggesting that Far1 and Sic1 are not required for Cip1-induced G1 arrest.

Cip1 is a CKI of all G1 cyclins. G1/S transition is controlled by Cdk1-G1 cyclins to activate downstream genes required for S phase initiation. We hypothesized that Cip1 overexpression may reduce Cdk1-G1 cyclin activity. To verify this, we induced G1 cyclin in the Cip1 overexpressed strain. Simultaneous induction of one of G1 cyclins in the Cip1 overexpressed strain partially rescued the Cip1-dependent slow growth through preventing G1 arrest (Fig. 1d). These results suggest that Cip1-mediated G1 arrest is due to the inhibitory targeting of Cip1 to Cdk1-G1 cyclin complexes and this arrest can be rescued by increasing G1 cyclin expression. This is consistent with a previous study showing that Cip1 inhibits Cdk1-Cln2 activity ${ }^{36}$. To further determine whether Cipl is a direct inhibitor of all three Cdk1-G1 cyclin complexes, we examined whether Cip1 could inhibit the kinase activity of all three Cdk1-G1 cyclins purified from yeast extracts. The presence of GST-Cip1 resulted in a prominent decrease of in vitro Cdk1-G1 cyclin activities (Fig. 1e). These findings indicate that Cip1 directly inhibits all Cdk1-G1 cyclins to block cell cycle.

CIP1 is expressed by $\mathrm{Mcm} 1$ with peak expression at G1. A large-scale gene expression profile hinted that CIP1 expression begins at the M/G1 transition ${ }^{37}$. To identify the precise expression pattern of CIP1 during the cell cycle, G1-synchronized cells were released, and CIP1 expression was monitored. The expression pattern of CIP1 mRNA was similar to a G1 cyclin, CLN2, which peaked at G1 periodically during cell cycle progression (Fig. 2a,b). Therefore, CIP1 is a cell cycle-regulated gene mainly expressed at G1.

$\mathrm{Mcm} 1$ is a transcription factor that modulates gene expression at M/G1 transition ${ }^{27-29}$. Examination of the upstream sequences revealed two putative ECB elements located at nt -355 to -340 
a

\begin{tabular}{|c|c|c|}
\hline \multicolumn{3}{|c|}{ Unbudded cells (\%) } \\
\hline Carbon source & Vector & pGAL1-CIP1 \\
\hline Raffinose & $30 \pm 5$ & $36 \pm 8$ \\
\hline Galactose & $35 \pm 9$ & $55 \pm 6$ \\
\hline Raffinose & \\
Galactose & 1n 2n
\end{tabular}

b

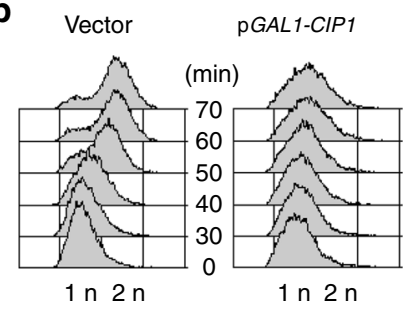

C

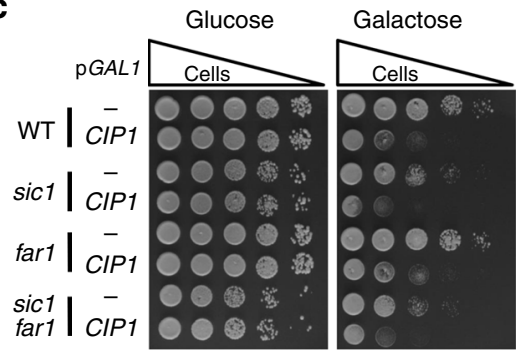

d
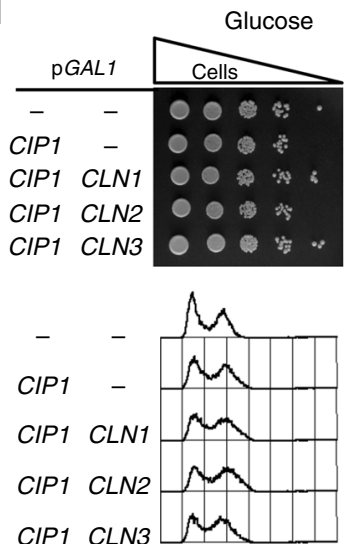

Galactose
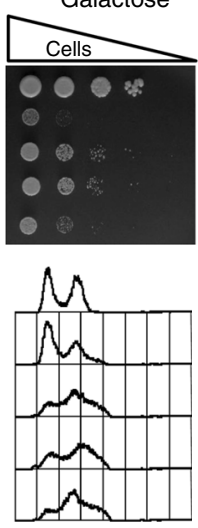

e

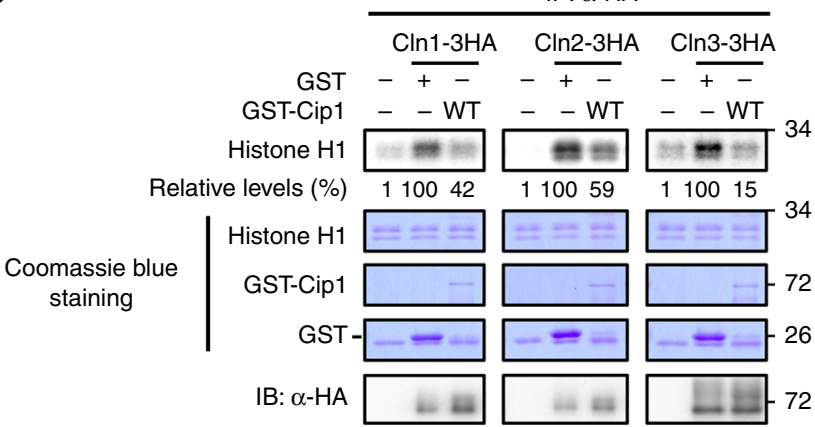

Fig. 1 Overexpression of CIP1 causes cell cycle arrest at G1 through inhibition of all Cdk1-G1 cyclin complexes. a Strains bearing either the empty vector or GAL1-CIP1 plasmid were pre-grown in $2 \%$ raffinose medium and then added $2 \%$ galactose to induce for $9 \mathrm{~h}$. The percentage of unbudded cells was scored. The values were given as mean \pm s.d. $(n=3)$. The DNA content was shown below by FACS analysis. b Strains with the empty vector or GAL1-CIP1 plasmids were first arrested at G1 phase by $\alpha$-factor in $2 \%$ raffinose culture. Cip1 was induced in the present of $2 \%$ galactose. The $\alpha$-factor was subsequently removed from the cultures, and samples were collected at the indicated time points for flow cytometry analysis. c Isogenic wild-type (WT), far1, and sic1 strains harboring the empty vector or GAL1-CIP1 plasmid were spotted in 10-fold diluted equal number of yeast on $2 \%$ glucose and $2 \%$ galactose plates. d Strains bearing the LEU2 empty vector or GAL1-CIP1 plasmids were co-transformed with the URA3 empty vector, GAL1-CLN1, GAL1-CLN2, or GAL1-CLN3 plasmids. Cells were spotted in 10 -fold diluted equal number of yeast on $2 \%$ glucose and $2 \%$ galactose plates. The FACS analysis of these strains is shown below. e The Cdk1-cyclins were immunoprecipitated from strains bearing the empty vector or plasmids with GAL1 driven 3HA tagged Cln1, Cln2, or Cln3 and incubated with recombinant Cip1. Histone $\mathrm{H} 1$ was used for the kinase substrate. The kinase activity was shown as the relative signals of ${ }^{32} \mathrm{P}$-labeled histone $\mathrm{H} 1$. The immune precipitates were probed with anti-HA antibody to detect the levels of purified cyclins. The uncropped Coomassie blue images are shown in Supplementary Fig. 11

and -281 to -266 relative to the initiation codon of Cip1 (Fig. 2c). To determine the contribution of these elements for CIP1 expression, we generated a chromosomal lac $Z$ reporter strain with the 500 bp upstream untranslated region of CIP1 fused to a lac $Z$ gene. The lac $Z$ expression was dramatically decreased when both ECB elements were mutated (Fig. 2d), indicating that the ECB elements contribute to $C I P 1$ transcription. We further generated mutations at the ECB elements of the endogenous CIP1 promoter. Northern blot analysis showed that the cell cycle-dependent CIP1 expression was compromised in the ecb $I+I I$ mutants (Fig. 2a,b). Next, we asked whether CIP1 is transcriptionally activated through direct $\mathrm{Mcm} 1$ binding at ECBs during M/G1 transition. Chromatin immunoprecipitation (ChIP) experiments demonstrated that there was an enrichment of Mcm1 binding at the CIP1 promoter. This binding was diminished in the ecb $I+I I$ mutants (Fig. 2e). The binding of $\mathrm{Mcm} 1$ peaked at $80 \mathrm{~min}$, the late mitosis stage, after cells released from G1 phase (Supplementary Fig. 1). Together these findings indicate that $\mathrm{Mcm} 1$ regulates $C I P 1$ expression periodically at M/G1 transition through directly binding to the CIP1 promoter.

CIP1 expression is induced upon stress. In natural environments, yeast encounters various stresses. Since CIP1 is a cell cycle inhibitor, we were curious whether the expression of
CIP 1 could be regulated by stresses. CIP1 mRNA expression was examined under several stresses including osmotic stress $(\mathrm{KCl})$, oxidative stress $\left(\mathrm{H}_{2} \mathrm{O}_{2}\right)$, carbon source starvation (low glucose) (Fig. 3a-e), DNA damage (MMS), heat shock $\left(37^{\circ} \mathrm{C}\right)$, and rapamycin treatment (Supplementary Fig. 2a-e). Northern analysis showed that CIP1 mRNA increased upon the treatment of osmotic stress, oxidative stress, carbon source starvation, and DNA damage. CIP1 expression reached the peak at 15 min after stress, and then gradually declined. Further investigation indicated that CIP1 expression increased at $5 \mathrm{~min}$ after $\mathrm{KCl}$ treatment (Supplementary Fig. 2f). However, spotting assays detected that cip1 deletion mutants were not sensitive to these stresses (Supplementary Fig. 3a, b), which could indicate redundancy. In any case, these results indicate that CIP1 may be involved in immediate stress response and cause temporary G1/S delay for adaptation to sudden changes of environment.

Stress-driven CIP1 expression depends on Msn2/4. Functional redundant transcription factors Msn2 and Msn4 can activate the transcription of stress response genes ${ }^{31-35}$. To examine whether the stress-induced CIP1 expression is controlled by Msn2/4, we monitored the CIP1 mRNA expression in wild-type and msn2 msn4 double mutants under stress treatment. CIP1 mRNA expression was not induced in msn2 msn4 cells (Fig. 3a-e). 
a

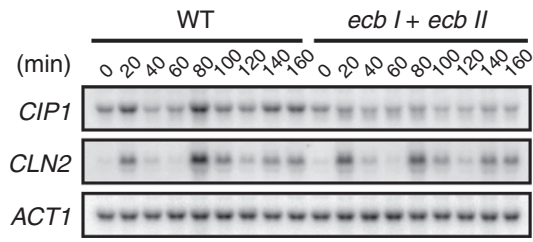

b

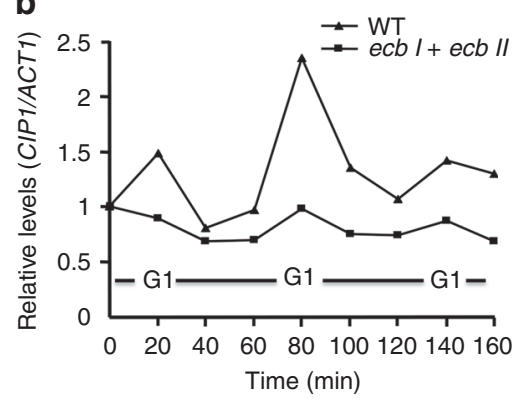

C

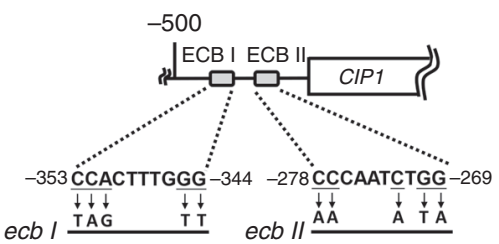

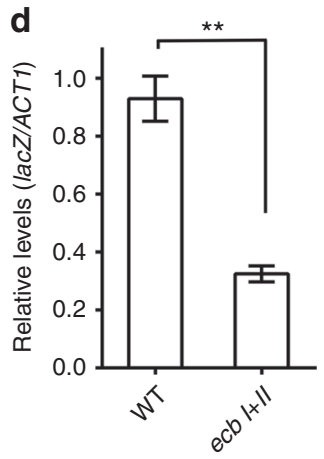

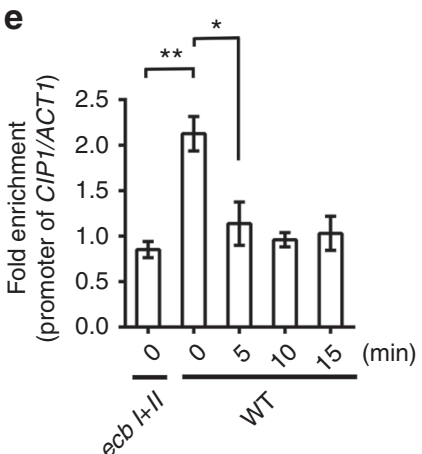

Fig. 2 CIP1 expression at M/G1 is mediated by Mcm1 through ECB elements on CIP1 promoter. a After synchronized cells had been released from G1 phase, total RNA was extracted from WT and ecb I+ecb // strains and analyzed by Northern blotting. $\mathbf{b}$ The quantitative results of the relative amount of CIP1 normalized to the internal control, ACT1, was shown. c Schematic diagram indicating two potential ECB elements on the CIP1 promoter. The mutation sites that destruct two ECB elements are shown below. $\mathbf{d}$ Reporter analysis was used to determine the contribution of ECB elements on the promoter activity of CIP1. The CIP1 promoter was placed at the upstream of the lacZ gene and then integrated into the yeast genome. To create the ecb I + II mutant, the ECB elements on the integrating plasmid were mutated before insertion. The lacZ expression was normalized to ACT1. The values were given as mean \pm s.d. $\left(n=3,{ }^{\star \star} P<0.01\right.$, Student's $t$-test, two tailed). e ChIP analysis of Mcm1 binding at CIP1 promoter and the times indicated the minutes after $0.5 \mathrm{M} \mathrm{KCl}$ treatment. The binding of Mcm1 on CIP1 promoter was normalized with U2 background. The values were given as mean \pm s.d. $\left(n=3\right.$, ${ }^{\star} P<0.05$, ${ }^{\star \star} P<0.01$, Student's t-test, two-tailed)

Furthermore, the ChIP results uncovered an enhanced Msn2 binding to the CIP1 promoter upon stress (Fig. $3 \mathrm{f}-\mathrm{h}$ ). Interestingly, association of $\mathrm{Mcm} 1$ at the CIP1 promoter decreased under osmostress (Fig. 2e).These results suggest that the stress-induced CIP1 expression is regulated by direct binding of Msn2/4 to the CIP1 promoter.

Several regulatory pathways suppress Msn2/4 activity. For example, inhibition of PKA or TOR pathway activates Msn2/4 ${ }^{38-40}$. Since CIP1 is a downstream target of Msn2/4, it might be spontaneously upregulated in the absence of Msn2/4 suppressor even without stresses. CIP1 expression was slightly increased in RAS, GPA, and TOR pathway blunted cells (Supplementary Fig. 4a). However, these mutants did not display an apparent growth defect upon osmostress (Supplementary Fig. 4b). These results suggest that RAS, GPA, and TOR pathways are relevant to Msn2/4-driven CIP1 expression. However, since the single abolishment of one of these pathways only slightly increases CIP1, it might not be sufficient to display a growth defect.

Upon stress, Msn2 and Msn4 translocate from cytoplasm into nucleus and bind to the STRE ${ }^{32,33}$. Two potential consensus STRE sites were observed at the upstream of CIP1 (Fig. 4a). To determine the contribution of these sequences for CIP1 expression, we generated a chromosomal lac $Z$ reporter strain containing mutations on both STRE sites of the CIP1 promoter. Under stress, the lac $Z$ expression was increased, but this upregulation was diminished when both STREs were mutated (Fig. 4b). Electrophoretic mobility shift assay (EMSA) further detected that recombinant Msn2 binds to these STREs directly (Fig. 4c). These results suggested that the STREs contribute to the transcriptional regulation of CIP1 promoter. Next, we directly created mutations of the STREs at the endogenous CIP1 promoter. Stress-induced CIP1 expression was diminished in stre $I$, stre II, and stre $I+I I$ mutant strains (Fig. 4d). The contribution of the two STREs was different: CIP1 mRNA expression was more significantly reduced in the stre $I$ strain and only additively decreased in stre $I+I I$ strain. These results demonstrate that both STREs are critical for stress-driven CIP1 expression when cells face environmental challenges. ChIP assay revealed that Msn2 directly binds to the STREs at the CIP1 promoter upon osmostress and this recruitment was inhibited in the stre $I+I I$ cells (Fig. 4e). Together these findings reveal that Msn2/Msn4 facilitate CIP1 expression under stresses.

Cip1 is phosphorylated under osmotic stress. Under osmotic stress, CIP1 mRNA was upregulated (Fig. 3b,e). We further examined Cip1 protein expression pattern under hyperosmotic stress and found that there was a gel mobility shift of Cip1 under hyperosmotic conditions. This shift was diminished by phosphatase treatment (Supplementary Fig. 5a). We wondered whether the phosphorylation of Cip1 is critical for yeast in adaptation upon osmostress. Initially, by using mass spectrometry (MS) analysis of purified GST-Cip1 from $\mathrm{KCl}-$ treated cells (Supplementary Fig. 5b), we identified that T65, T69, and T73 (hereafter called 3T) were phosphorylated under osmostress (Supplementary Fig. 5c). To determine whether the phosphorylation is critical for cells to adapt to the osmostress, we mutated T65, T69, and T73 from threonine to alanine to mimic non-phosphorylated Cip1, or to glutamate in an attempt to 
a

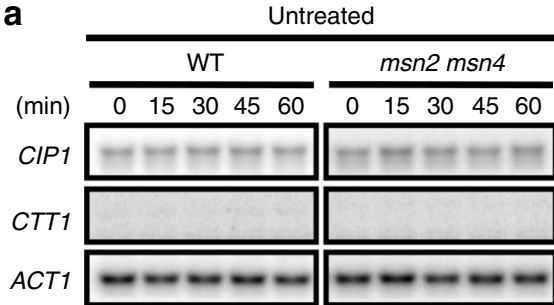

C

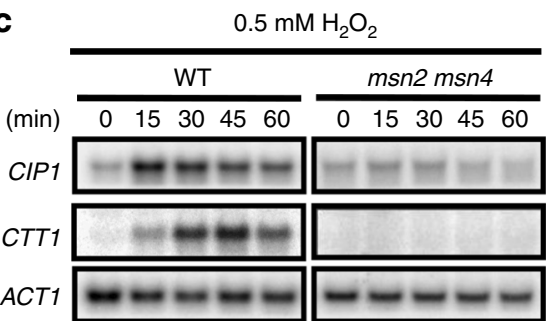

b

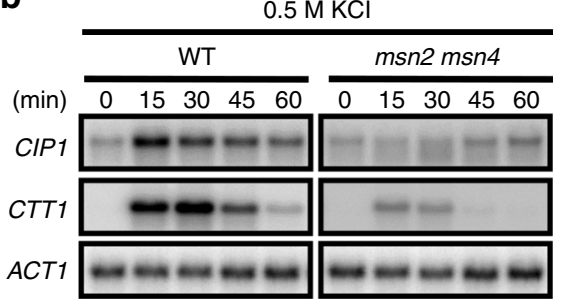

d

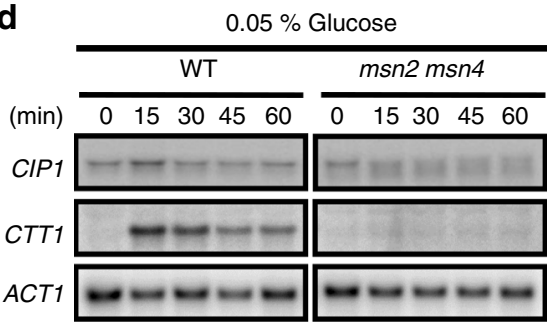

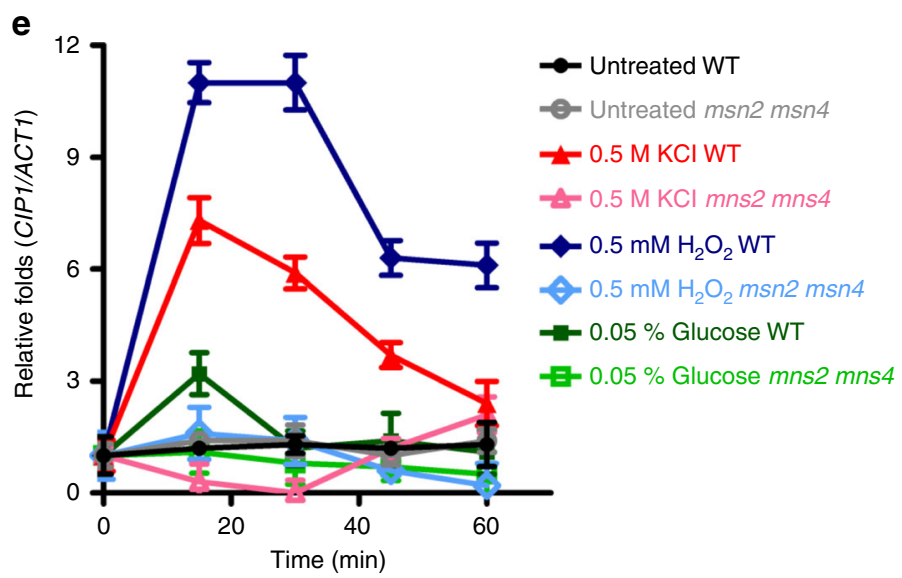
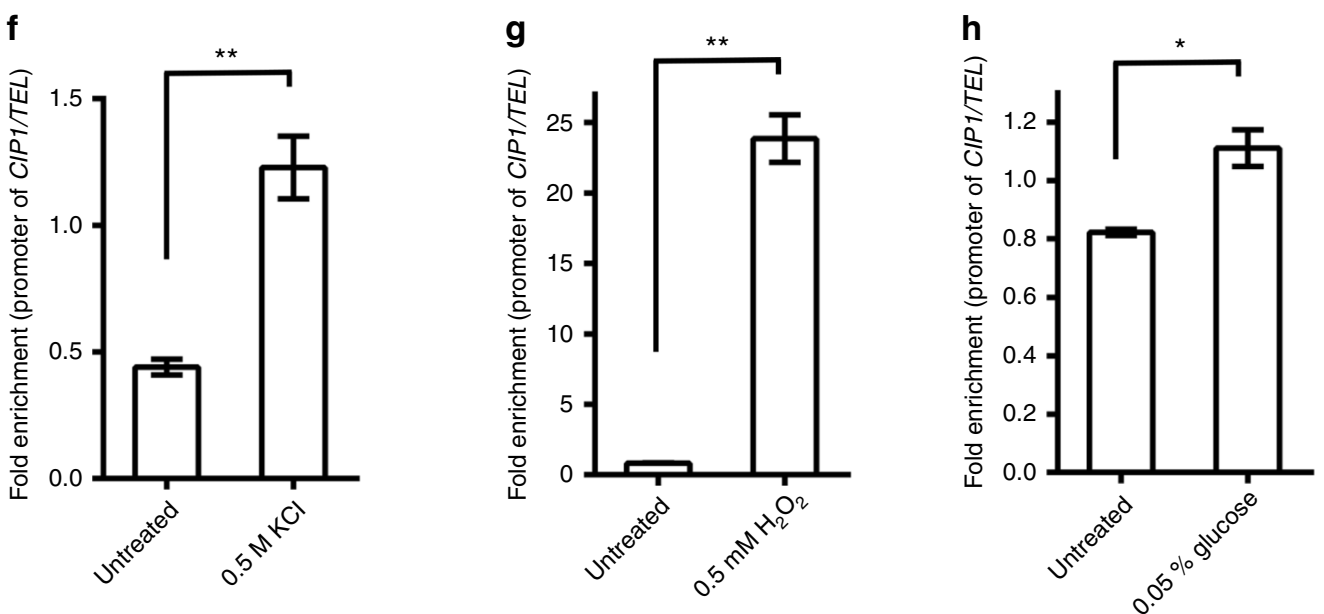

Fig. $3 \mathrm{CIP1}$ expression is induced under several stresses. Yeast cells were treated with various stresses including $\mathbf{a}$ untreated, $\mathbf{b}$ osmotic stress $(0.5 \mathrm{M} \mathrm{KCl})$, c oxidative stress $\left(0.5 \mathrm{mM} \mathrm{H}_{2} \mathrm{O}_{2}\right)$, and $\mathbf{d}$ carbon source starvation $(0.05 \%$ glucose). Samples were collected at the indicated time points after stress treatment. Total RNA was analyzed by Northern blotting and hybridized with indicated probes. CTT1 referred to the positive control of these stresses, and ACT1 was used as a loading control. e The band intensities displayed in the broken-line graph of each panel were quantified using Image J, normalized relative to respective internal controls, and expressed as the ratio of the CIP1 levels to the time point 0 at the beginning of each stress treatments. The values were given as mean \pm s.d. $(n=3)$. mRNA expression was considered to be upregulated when relative fold exceeds 2 . f-h ChIP analysis of Msn2 binding at the CIP1 promoter under $\mathbf{f}$ osmotic stress $(0.5 \mathrm{M} \mathrm{KCl}), \mathbf{g}$ oxidative stress $\left(0.5 \mathrm{mM} \mathrm{H}_{2} \mathrm{O}_{2}\right)$, and $\mathbf{h}$ carbon source starvation (0.05\% glucose). The binding of Msn2 at the CIP1 promoter was normalized by that at the TEL sequence. The values are given as mean \pm s.d. $\left(n=3,{ }^{\star} P<0.05,{ }^{\star \star} P<0.01\right.$, Student's $t$-test, two-tailed) 
a

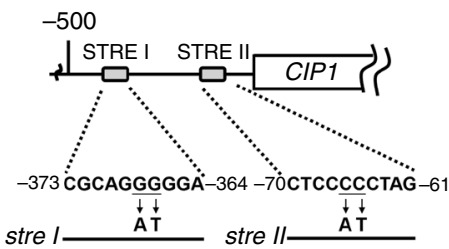

b

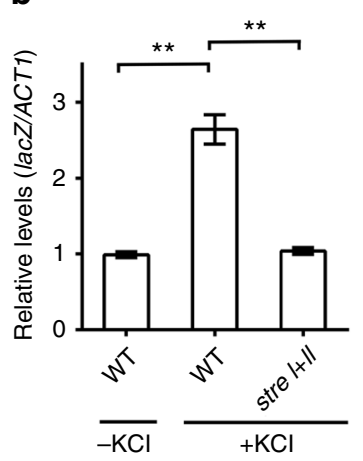

C

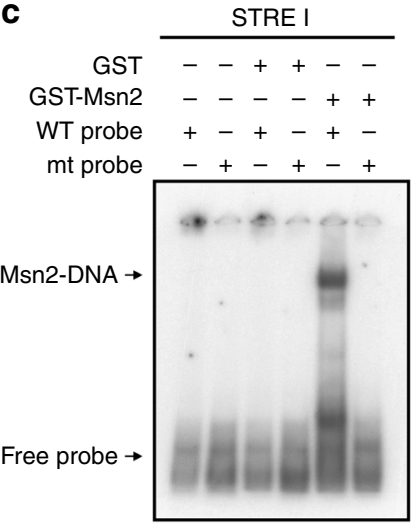

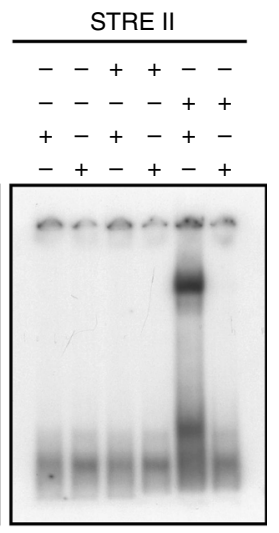

d
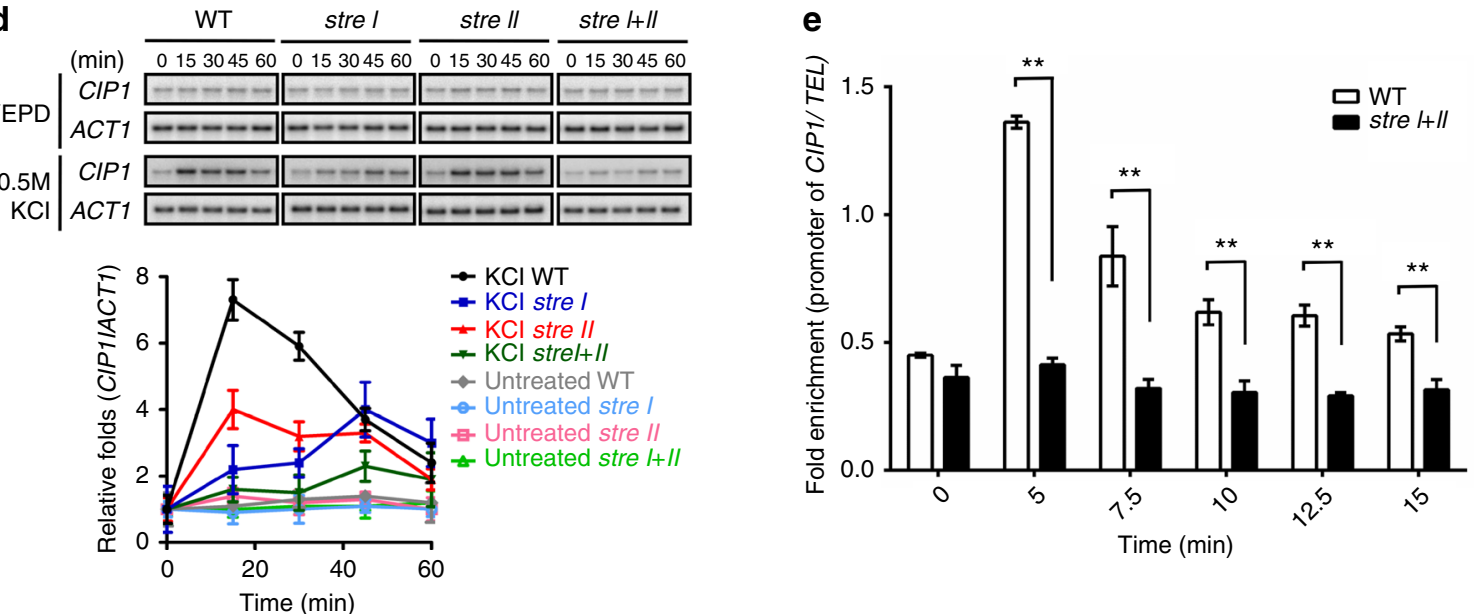

Fig. 4 Msn2/4 regulate CIP1 expression through two STRE binding sites. a Schematic diagram indicates two putative STREs at the CIP1 promoter. The mutation sites that destruct STREs are shown below. $\mathbf{b}$ Reporter analysis was used to determine the contribution of STRE on the promoter activity of CIP1. Osmotic stress was induced by $0.5 \mathrm{M} \mathrm{KCl}$. The lacZ expression was normalized by ACT1 expression. The values are given as mean \pm s.d. $\left(n=3,{ }^{\star \star} P<0.01\right.$, Student's t-test, two-tailed). c EMSA was performed with STRE I, mutant stre I, STRE II or mutant stre II-radiolabeled double-stranded oligonucleotides. Recombinant GST or GST-Msn2(401-704) proteins were subjected to EMSA. d Top: Northern blot analysis of CIP1 expression in WT and STRE mutant strains (stre I, stre II, and stre I+II) under osmotic stress. Bottom: The band intensities displayed in the broken-line graph was quantified using Image J, normalized relative to respective internal controls, and expressed as the ratio of the CIP1 levels to the time point 0 at the beginning of $0.5 \mathrm{M}$ $\mathrm{KCl}$ treatments. The values were given as mean \pm s.d. $(n=3)$. e ChIP analysis of Msn2 binding at CIP1 promoter under osmotic stress at indicated times. The binding of Msn2 at the CIP1 promoter was normalized by the TEL background. The values are given as mean \pm s.d. $\left(n=3,{ }^{\star \star} P<0.01\right.$, Student's t-test, two-tailed)

mimic phosphorylated Cip1. The spotting assay indicated that overexpressed CIP1, but not cip1-3TA, caused growth inhibition (Fig. 5a). Overexpressed Cip1-3TE did not delay cell growth as the wild-type Cip1 (Fig. 5a), implying that the Cip1-3TE mutation may not be sufficient reflection of the wild-type function. Moreover, to identify the contribution of each phosphorylation site on growth, T65, T69, and T73 were double mutated with each other to alanine. Double mutations of T65A and T73A were sufficient to lose the ability of growth inhibition, but not the other combinations (Supplementary Fig. 6a), suggesting that the phosphorylations of T65 and T73 are necessary for Cip1-induced growth inhibition.

Hog1 phosphorylates Cip1 T65 and T73 under osmotic stress. Hog1 is the effector protein kinase of the high osmolarity glycerol (HOG) pathway and transiently translocates from the cytosol to nucleus where it controls gene expression during hyperosmoadaption $^{41,42}$. Under the hyperosmotic condition, Hog1 is essential for cell survival through regulating gene expression, glycerol accumulation, signal fidelity and cell cycle arrest ${ }^{43}, 44$.
Since the T65, T69 and T73 of Cip1 were phosphorylated under osmotic stress, we speculated Hogl as the potential kinase. To determine whether Hog1 directly phosphorylates Cip1, in vitro kinase assay was conducted using purified recombinant Hog1, the Hog1 activator Pbs2 MAPKK, and Cip1. Using Sic1 as a positive control $^{45}$, the in vitro kinase assay showed that Hog1 directly phosphorylated Cip1, but not Cip1-3TA. Similar inhibition was observed when a Hog1 inhibitor (SB203580) was included (Fig. 5b), suggesting that Cip1 is a direct target of Hog1.

We further generated phosphor-specific antibodies (Supplementary Fig. $6 \mathrm{~b}$ and c) to detect the phosphorylation states of Cip1 under osmotic stress. A prompt phosphorylation of Cip1 was observed within $5 \mathrm{~min}$ after hyperosmotic stress, and the phosphorylations were then gradually decreased at the following time points (Fig. 5c). In hog1 cells, Cip1 T65 and T73 were not phosphorylated under osmotic stress (Fig. 5c), suggesting that these phosphorylations require Hog1. Together, these results demonstrate that Hog1 directly phosphorylates Cip1 upon hyperosmotic stress. The status of Cip1 phosphorylation during cell cycle progression was also monitored. There was no detectable Cip1 T65 and T73 phosphorylation during the cell 
a

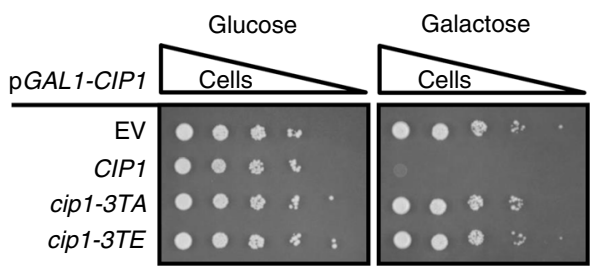

b

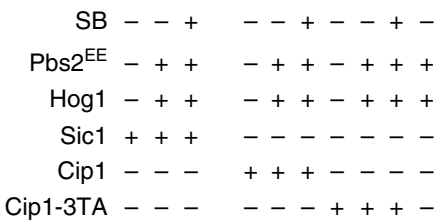

(kDa)

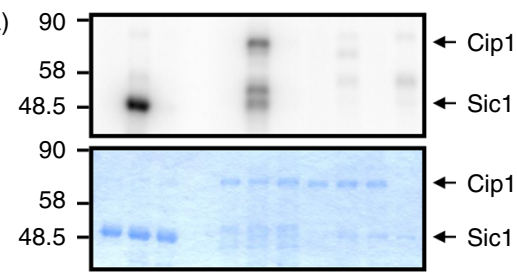

$\mathbf{C}$

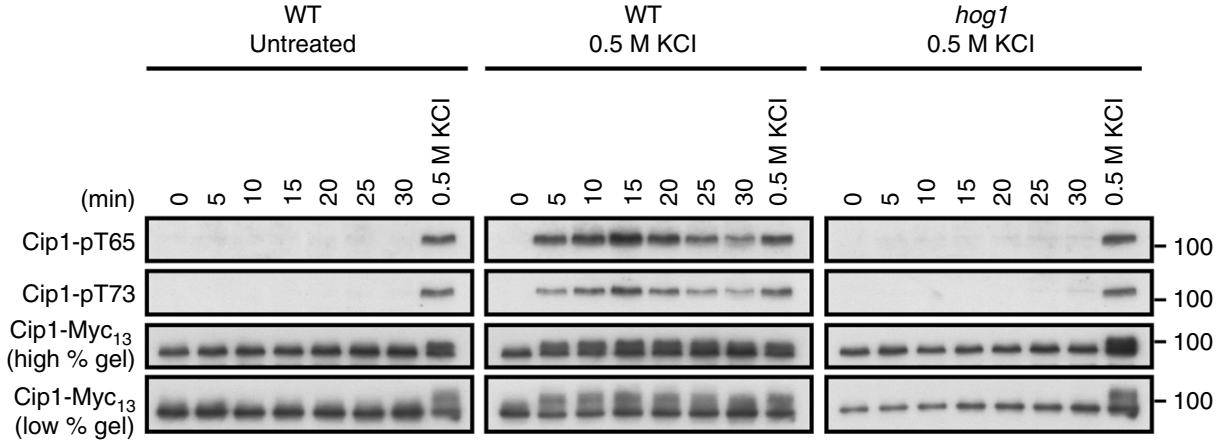

Fig. 5 Cip1 is phosphorylated by Hog1 kinase directly under osmotic stress. a Isogenic WT strains bearing empty vector, GAL1-CIP1, GAL1-cip1-3TA, or GAL1cip1-3TE plasmid were spotted in 10-fold diluted equal number of yeast on $2 \%$ glucose and $2 \%$ galactose plates. b Recombinant GST-Sic1, GST-Cip1, and GST-Cip1-3TA were employed as substrates for in vitro kinase assay. GST-Hog1 was activated by incubation with GST-Pbs2 ${ }^{\mathrm{EE}}$ for 30 min at $30{ }^{\circ} \mathrm{C}$. Activated Hog1 was added to the substrate and incubated at $30^{\circ} \mathrm{C}$ for $30 \mathrm{~min}$ in the presence of $32 \mathrm{P}$-ATP. Proteins were resolved by SDS-PAGE and stained with Coomassie blue (bottom), and phosphorylated proteins were detected by autoradiography (top). Sic1 was included as a positive control; SB203580 was used to selectively inactivate Hog1. c Cip1 phosphorylations in WT cells, WT cells treated with $0.5 \mathrm{M} \mathrm{KCl}$, and hog1 cells treated with $0.5 \mathrm{M}$ $\mathrm{KCl}$ were detected by phosphor-specific antibodies, pT65 and pT73, and total Cip1 was served as a loading control. The mobility shift of phosphorylated Cip1 was shown in low percentage gel

cycle (Supplementary Fig. 6d). A previous study showed that Cdk1-Clb complex phosphorylates $\mathrm{Cip}^{36}$ and both Cdk1 and Hog1 share the same consensus phosphorylation sites (S/T-P). To further understand whether the phosphorylations of T65 and T73 were cell cycle-dependent, the cdc28-as1 strain was treated with 4-amino-1-tert-butyl-3-(1'-naphthylmethyl) pyrazolo [3,4-d] pyrimidine(1-NM PP1) to block Cdk1 activity under osmotic stress ${ }^{46}$. Inhibition of Cdk1 activity displayed no influence on osmostress-induced Cip1 phosphorylations (Supplementary Fig. 6e). These results indicate that Cip1-3T phosphorylation is only induced under osmostress, but not in regular mitotic cycle.

Cip1 contributes to osmostress-induced transitory G1 delay. Osmotic stress causes transient G1 delay of cells, which leads to retarded budding formation ${ }^{7}$. CIP1 mRNA is upregulated upon hyperosmotic stress (Fig. 3b,e) and Cip1 overexpression leads to cell cycle arrest (Fig. 1b). To determine whether Cip1 contributes to transient G1 arrest under hyperosmotic conditions, the fraction of budded cells of cip1 strains in exponentially growing cultures was measured. Consistent with previous findings ${ }^{p}$, the lowest budding index of wild-type cells was detected at 40 min after $\mathrm{KCl}$ treatment. The budding index of the cip1 cells at $40 \mathrm{~min}$ was higher than that of wild-type cells (Fig. 6a), suggesting that Cip1 contributes to osmotic stress-induced transitory cell cycle arrest. The budding index of cip1-3TA cells was similar to that of cip1 cells; both are higher than wild-type cells, suggesting that Hog1 controls Cip1-dependent cell cycle delay under osmotic stress.

Sicl was reported as a CKI that causes G1 arrest under osmotic stress ${ }^{45}$. To evaluate the contribution of Sicl and Cip1 in response to osmostress, the budding indexes of sic1 and sic1 cip1 strains were also monitored. Deletion of SIC1 gave rise to more budded cells under untreated condition (Fig. 6a, time 0), suggesting that SIC1 plays an essential role in G1 in the regular mitotic cycle. At $40 \mathrm{~min}$ after $\mathrm{KCl}$ treatment, the change of budding index of sic1 cells (77.6 to $60.3 \%)$ was almost the same as that of cip1 cells (65.8 to $47.8 \%$; Fig. 6a). Surprisingly, the budding index was much higher in sic1 cip1 double mutants than either sic1 or cip1 single mutants (Fig. 6a), suggesting a redundant role of these CKIs in the acceleration of S-phase entry. We observed that $\alpha$-factor treatment downregulates Cip1 expression (Supplementary Fig. 6d). To further investigate the biological functions of Cip1 but avoid the disturbance of $\alpha$-factor treatment, we elutriated wild-type, cip1, sic1, and cip1 sic1 strains to analyze the cell cycle progression under osmotic stress of newborn cells. The FACS analysis showed no difference between wild-type and cip 1 cells, while the loss of CIP1 in sic1 cells further accelerated cell cycle progression at $120 \mathrm{~min}$ after osmotic stress treatment (Fig. 6b). Also, CIP1 single deleted cells did not exhibit a stresssensitive phenotype compared to wild-type cells; however, deletion of CIP1 further augmented the stress-sensitive phenotype in sic1 cells (Fig. 6c; Supplementary Fig. 3b). These results revealed that Cip1 collaborates with Sicl in osmotic stress-induced G1 cell cycle arrest, implying that Sic1 and Cip1 


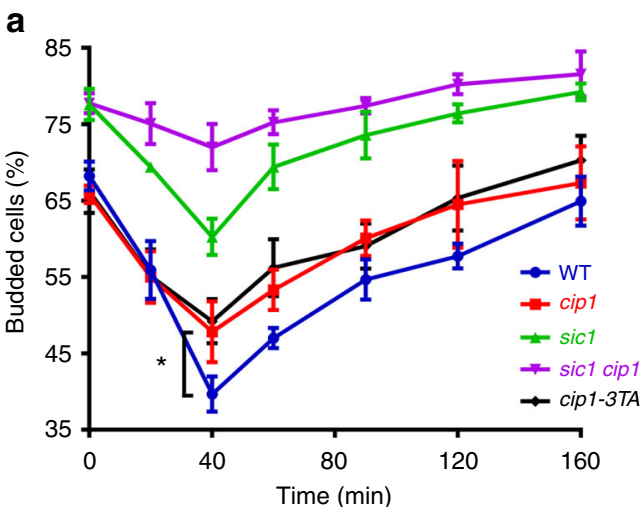

C

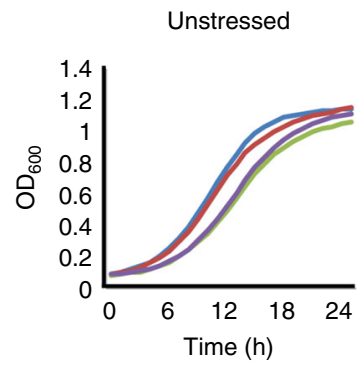

b

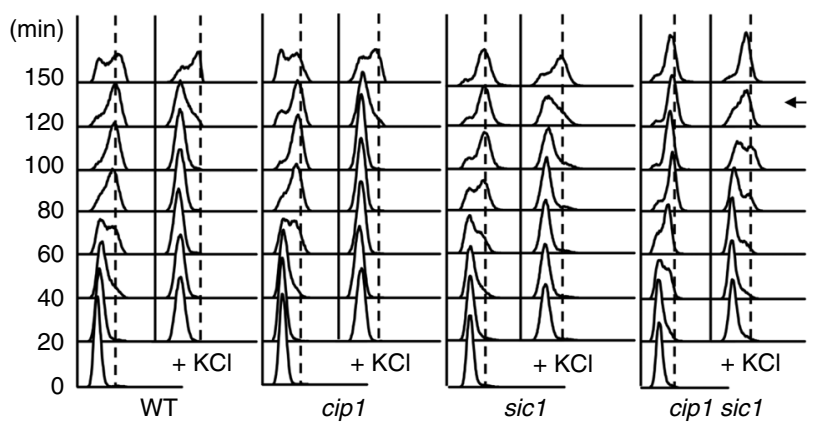

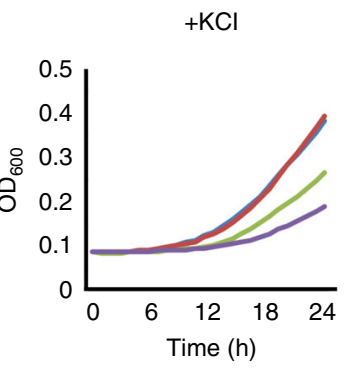

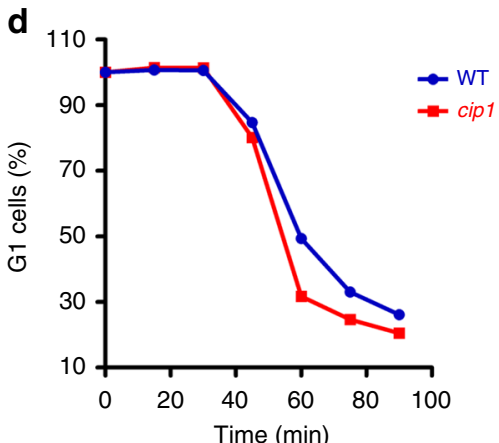

Fig. 6 The phosphorylation of Cip1 is responsible for hyperosmotic stress-induced transitory G1 delay. a Budding index of WT, cip1, cip1-3TA, sic1, and sic1 cip1 strains under treatment of $0.5 \mathrm{M} \mathrm{KCl}$. The percentage of budded cells were given as mean \pm s.d. $\left(n=3,{ }^{\star} P<0.05\right.$, Student's $t$-test, two-tailed). b WT, cip1, sic1 and cip1 sic1 strains were elutriated in YEP, raffinose. Newborn cells were collected and recovered in YEPD at $25^{\circ} \mathrm{C}$. At time 0, cultures were subjected to or not to osmostress $(0.5 \mathrm{M} \mathrm{KCl})$. Cell cycle progression was monitored by FACS analysis for 150 min after stress. An arrow indicates the timing when the G1-S transition speeds up in cip 1 sic1 cells. $\mathbf{c}$ Exponentially growing cells were diluted to $\mathrm{OD}_{600}=0.1$ and grown at $30{ }^{\circ} \mathrm{C}$ in minimal media. Osmosensitivity was tested in the presence of $1.2 \mathrm{M} \mathrm{KCl}$. OD measurements were estimated every hour for $25 \mathrm{~h}$ in a 96 -well plate using Synergy $\mathrm{H} 1 \mathrm{Multi}-$ Mode Reader. $\mathbf{d}$ In WT and cip1 strains, $\alpha$-factor synchronized G1 cells were released in $0.5 \mathrm{M} \mathrm{KCI} \mathrm{YEPD} \mathrm{medium} \mathrm{at} 24^{\circ} \mathrm{C}$. At 15 min intervals, samples were collected, and $\alpha$-factor/nocodazole trap assay was performed. More than 300 cells showing mating projections (G1 cells) or buds (post-G1 cells) were counted for each time point, and the experiment was repeated three times

are functionally redundant proteins when cells encounter environmental challenges.

Although the results of budding index indicate that Cip1 contributes to osmotic stress-induced transitory cell cycle arrest, the regular FACS analysis of wild-type and cip1 cells showed no obvious difference of cell cycle progression under the treatment of osmotic stress (Fig. 6b; Supplementary Fig. 7a). To further distinguish early S phase from G1 arrest, which might be insensitive to FACS, we analyzed the wild-type and cip1 cells using an $\alpha$-factor/nocodazole trap assay. G1 synchronized wild-type and cip1 cells were released in medium containing $0.5 \mathrm{M} \mathrm{KCl}$. At $15 \mathrm{~min}$ intervals, cells were transferred to trapping medium ( $\alpha$-factor and nocodazole) and incubated for $90 \mathrm{~min}$. Those cells that remain in G1 were sensitive to $\alpha$-factor and form mating projections; in contrast to any cells that left G1 passing START were arrested in nocodazole at G2/M as large budded cells. Performing this assay under osmotic stress revealed that cip1 cells exited G1 earlier than wild-type cells (Fig. 6d). Altogether, these results indicate that Cip1 regulates osmotic stress-induced G1 delay through Hog1-mediated phosphorylation.

Cip1 phosphorylation may enhance the binding to Cdk1-Clns. Cip1-dependent slow growth can be partially rescued through simultaneous induction of G1 cyclin (Fig. 1d), implying that Cip1-mediated G1 arrest is probably due to the inhibitory targeting of Cip1 to Cdk1-G1 cyclin complexes. A previous study found that Cip1 physically interacts with the Cdk1-Cln2 complex $^{36}$. To investigate the physical interaction of Cip1 with the three Cdk1-G1 cyclin complexes, we performed co-immunoprecipitation to determine their associations. The Myc-tagged Cip1 and $\mathrm{HA}_{3}$-tagged $\mathrm{Cln} 1, \mathrm{Cln} 2$, or $\mathrm{Cln} 3$ proteins were overexpressed under the GAL1 promoter. Under osmotic stress, Cip1 exhibited higher binding affinity to each of the G1 cyclins (Fig. 7a). These interactions decreased in the cip1-3TA mutant (Fig. 7b).Collectively, these data suggest that Cip1 can interact with all Cdk1-G1 cyclin complexes and the Hog1-mediated phosphorylation might strengthen the binding affinity (Fig. 7c).

Cip1 impedes G1 through inhibiting Cdk1-Cln3 activity. Among three G1 cyclins, Cln3 is the most upstream regulatory G1 cyclin before the START. The Cdk1-Cln3 phosphorylates Whi5 for the activation of SBF, which induces specific G1-S transition genes ${ }^{16}$. We therefore speculated that $\mathrm{Cdk} 1-\mathrm{Cln} 3$ may be the most upstream target of Cip1 at early G1. To confirm whether Cip1 delays early G1 phase through inhibiting $\mathrm{Cdk} 1-\mathrm{Cln} 3$, the yeast strains bearing the empty vector or GAL1-CIP1 overexpressing plasmid were synchronized at G1 and Whi5 phosphorylation was monitored after release (Supplementary Fig. 7b). In cells carrying an empty vector, phosphorylated Whi5 appeared and peaked at $75 \mathrm{~min}$ after releasing (Fig. $7 \mathrm{~d}$ ). 
a

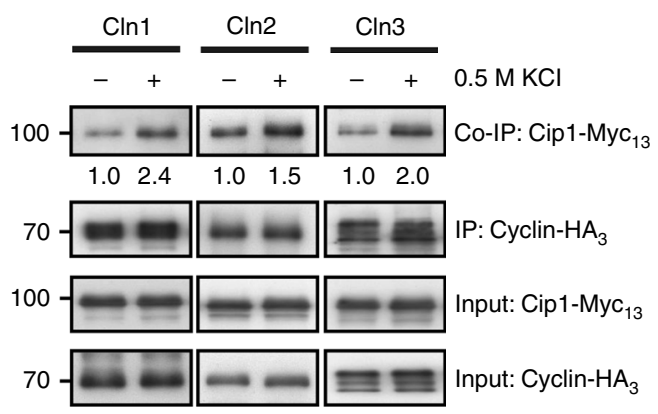

b

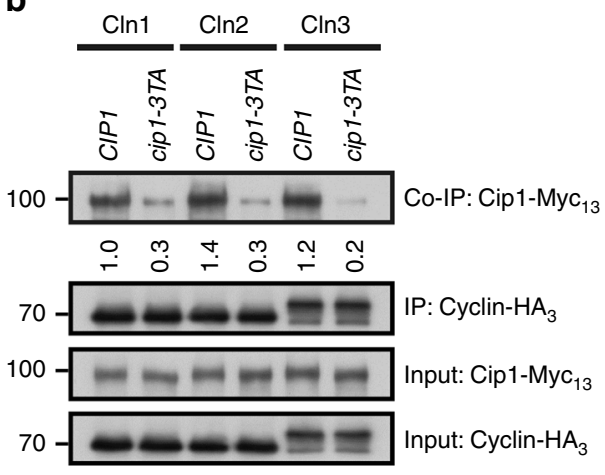

C

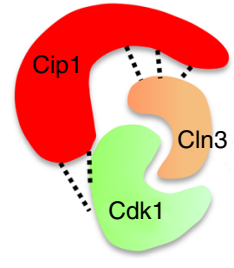

Loosely bound

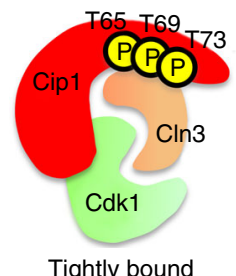

d
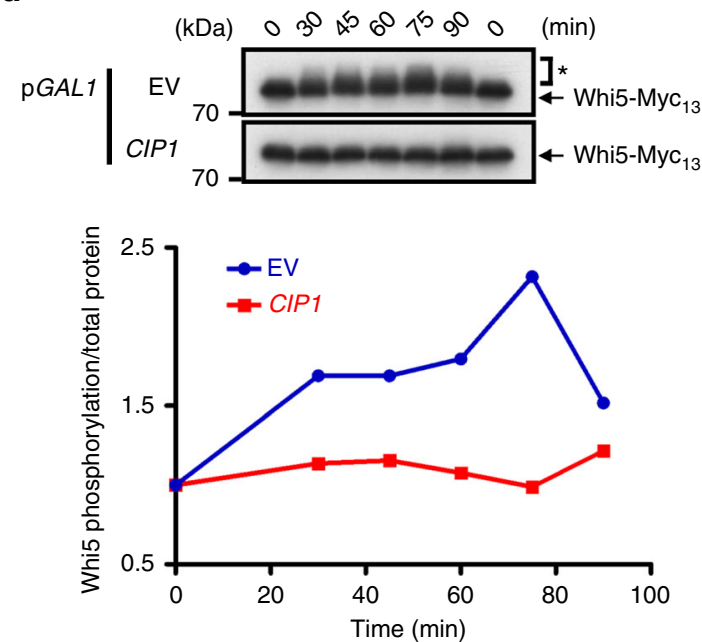

f

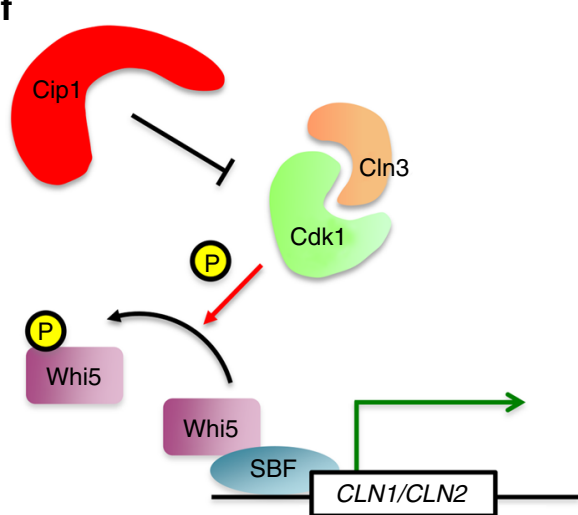

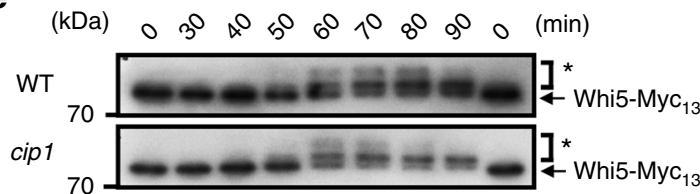

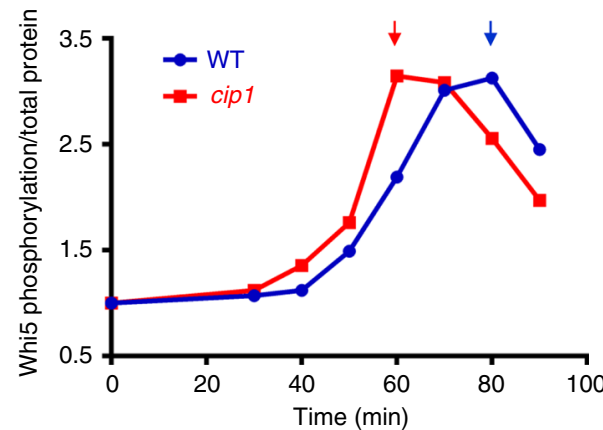

g

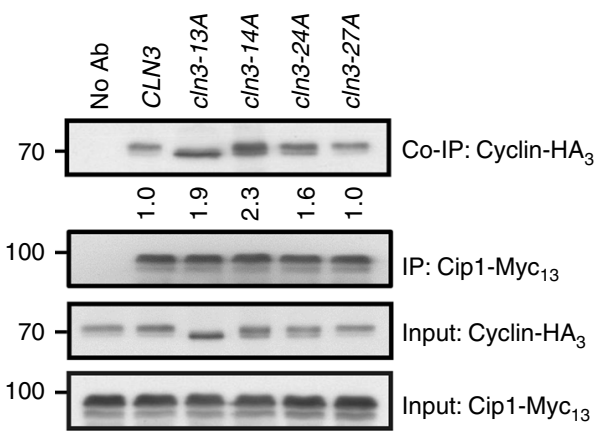

However, this phenotype was not observed in CIP1-overexpressing cells. Under osmotic stress, yeast cells arrest in G1 transiently ${ }^{7}$. To determine whether Cipl could transiently inhibit Cdk1-Cln3 activity under hyperosmotic stress, the timing of appearance of phosphorylated Whi5 in wild-type and cip1 cells were monitored. $\alpha$-factor released cell cycle progression was analyzed by FACS analysis (Supplementary Fig. 7c). After $\mathrm{KCl}$ treatment, the Whi5 phosphorylation in cip1 cells peaked at 60 min, which was $20 \mathrm{~min}$ faster than that in wild-type cells (Fig. 7e), suggesting that Cip1 inhibits Cdk1-Cln3 activity and thereby prevents Whi5 phosphorylation. The unphosphorylated Whi5 suppresses SBF and causes cell cycle arrest (Fig. 7f).
Cdk1-Cln3 interaction prevents Cip1-Cln3 binding. We next asked whether the Cdk1-Cln3 interaction is crucial for Cip1 function. Several sites on Cln3 are critical for its interaction with $\mathrm{Cdk} 1^{47}$. To this end, the interactions between Cip1 and wild-type Cln3, three Cln3 mutants, Cln3-13A (K163D166A), Cln3-14A (D166R170A), and Cln3-24A (K357K359A), which are defective in Cdk1 interaction, and a non-related control mutant, Cln3-27A (E386E387AR390A), which maintains the Cdk1-Cln3 interaction, were examined. In contrast to wild-type $\mathrm{Cln} 3$ and a nonrelated control (Cln3-27A), the interaction between Cip1 and $\mathrm{Cln} 3$ increased in the Cdk1 interaction-defective $\operatorname{cln} 3$ mutants, $\operatorname{cln} 3-13 A, \ln 3-14 A$, and $\ln 3-24 A$ (Fig. $7 \mathrm{~g}$ ). These results revealed 
that Cip1 exhibits a stronger association with Cdk1 interactiondefective Cln3, implying an enhanced binding of Cip1 to the free form of Cln3. This Cip1-Cln3 binding might block Cdk1 to form a complex with $\mathrm{Cln} 3$ and therefore inhibit the kinase activity.

Cip1 localizes to the nucleus upon osmotic stress. Our findings indicate that Cip1 can interact with all Cdk1-G1 cyclin complexes to inhibit cell cycle progression and the Hog1-mediated phosphorylation on Cip1 strengthens the Cip1-Cdk1-Cln interaction. Under osmotic shock, Hog1 translocates transiently to the nucleus ${ }^{48}$. To investigate the localization of Cip1 under osmotic stress, a C-terminal GFP-fused Cip1 was overexpressed in wild-type and hogl cells. Fluorescence microscope examination revealed that Cip1 was localized throughout the cytoplasm and nucleus in unstressed cells. When cells were exposed to osmotic shock, the fluorescence signal of Cipl accumulated within the nucleus. In hog1 cells, Cip1 was still able to accumulate in the nucleus under osmotic stress (Supplementary Fig. 8). These data suggest that Cip1 localizes to the nucleus after osmotic stress.

Human p21 expression stimulates G1 arrest in yeast cells. Cip1 is induced by stress and activated by Hog1 for G1 arrest. The mammalian homolog of Hog1, p38, also phosphorylates downstream stress-induced p21. Since Cip1 contains a conserved CDK-binding motif found in human $\mathrm{p} 21^{36}$, it drew our attention that Cip1 might be a homologue of human p21. The spotting assay showed that overexpression of p21 resulted in inhibition of yeast growth. This growth retardation was caused by G1 arrest as determined by FACS analysis (Supplementary Fig. 9). The functional similarity of p21 to Cip1 implies that Cip1 may be a homolog to human p21.

\section{Discussion}

Cip1 was recently characterized as a new G1 CKI which associates with $\mathrm{Cdk} 1$ and $\mathrm{Cln} 2^{36}$. However, its detailed regulatory mechanisms to control cell cycle progression are poorly understood. Our studies uncovered that Cip1 not only inhibits Cdk1-Cln2 but all Cdk1-Cln complexes to arrest G1 progression. $C I P 1$ is specifically transcribed from $\mathrm{M} / \mathrm{G} 1$ to late $\mathrm{G} 1$, and its expression is modulated by the upstream activating sequence in an $\mathrm{Mcm} 1$-dependent manner in vivo at $\mathrm{M} / \mathrm{G} 1$. In the previous study, CIP1 was also found to be regulated by Mbp1, a G1 transcription factor. We infer that $\mathrm{Mcm} 1$ binds to Cip1 promoter at M/G1 stage and the early expressed Cip1 mainly regulates Cdk1-Cln3 at early G1. Mbp1 subsequently induces CIP1 expression, and the $\mathrm{Cdk} 1-\mathrm{Cln} 1 / \mathrm{Cln} 2$ are the primary targets of Cip1 at late G1 (Supplementary Fig. 10).
Our studies also demonstrated that CIP1 is upregulated by various stresses, which is mediated by the key regulators of the stress-responsive genes, Msn2/4. The STREs at the CIP1 promoter are essential for the binding of $\mathrm{Msn} 2 / 4$ to promote CIP1 expression under stress. In wild environments, organisms require the proper response and protective mechanism to adapt to stress. There are many stress response genes expressed to help cells to overcome the external challenge. Cip1 may be the stress response gene to regulate G1/S transition. Under hyperosmotic condition, cip1 cells escape from transitory G1 arrest and display higher budding index. $\alpha$-factor/nocodazole assay also reveals that cip1 cells exit G1 faster under osmotic stress. Cells need time to activate a particular set of genes for building up proper cellular responses to adapt to stress and recover from damage. Cip1 may be the regulatory protein responsible for the G1 arrest, which ensures cells to make the appropriate response to overcome the challenge before $\mathrm{S}$ phase initiation. Even though CIP1 is induced in response to multiple stresses, CIP1-deleted cells are not sensitive to these stresses. It is likely that Cip1 causes transient G1/S delay, in collaboration with Sic1, for adaptation to sudden environmental changes, but later on the delay might be recovered in the following cell cycle stages. Furthermore, Hog1-mediated Whi5 and Msa1 phosphorylations also inhibit G1 cyclins expression to control adequate passage through START upon osmostress $^{17}$. Therefore, multiple regulatory pathways at G1/S might accumulatively delay G1 for cells to cope with stress.

The osmotic stress responses are mostly regulated by the SAPK signaling pathway ${ }^{49}$. Hog 1 is the effector kinase of this pathway, which controls gene expression during hyperosmoadaption ${ }^{42-44}$. Hog1 phosphorylates Cip1 T65, T69, and T73 upon hyperosmotic stress. However, the phosphorylation of Cipl could only be detected from the TCA-treated total lysates, due to the significant instability of phosphorylated Cip1 immediately after cell breakage. The Hog1-mediated phosphorylation might increase the interaction with Cdk1-G1 cyclins to induce transitory cell cycle arrest. All these findings establish that Cip1-mediated cell cycle arrest occurs through the inhibitory targeting to Cdk1-G1 cyclins (Fig. 8). Cip1-3TA loses the inhibitory phenotype of Cip1; however, the phospho-mimetic Cip1 mutant does not restore the wild-type function. It is not unusual that phosphomimetic mutants cannot completely simulate the structures of phospho-serine and phospho-threonine, given the fact that the negative charge introduced by glutamate or aspartate substitution $(-1)$ does not match with that of the phosphorylated residue $(-2)$ at physiological $\mathrm{pH}^{50}$. The inhibitory association of Cip1 with the most upstream regulatory Cdk1-G1 cyclin, Cdk1-Cln3, inhibits Whi5 phosphorylation. Without CDK-mediated phosphorylation, Whi5 inactivates SBF and results in temporary G1/S delay.

Fig. 7 Cip1 regulates G1/S progression by inhibiting Cdk1-Cln3 complex activity. a Co-immunoprecipitation indicated that osmotic stress augments the Cip1-G1 cyclin interactions. Immunoprecipitation was conducted using an anti-HA antibody to pull-down GAL1 promoter-driven cyclins. The levels of signal compared with that of the untreated cells are shown below. b Co-immunoprecipitation indicated that Cip1 phosphorylation site mutation impairs the Cip1-G1 cyclin interactions. c A cartoon describes the function of phosphorylation of Cip1. The phosphorylation of Cip1 on T65, T69, and T73 increases the binding affinity between Cip1 and Cdk1-Cln3 complex. d The Myc 13 -tagged WHI5 strain harboring empty vector, GAL1-CIP1, or GAL1-cip1-3TA was synchronized at G1 by $\alpha$-factor in $2 \%$ raffinose. Cip1 was induced in the present of $2 \%$ galactose. The $\alpha$-factor was subsequently removed from the cultures and samples were collected at 15 -min intervals for $90 \mathrm{~min}$ at $24^{\circ} \mathrm{C}$. Whi5 was detected by western blotting using an antibody against Myc, and the phosphorylated Whi5 was indicated by an asterisk $\left({ }^{*}\right)$. The relative band intensities of phosphorylated Whi5 to total Whi5 was quantified and expressed as the ratio of the phosphorylated Whi5 levels to the time point 0. e The chromosomal WHI5 was tagged with Myc 13 in WT and cip1 cells. Cells were synchronized at $\mathrm{G} 1$ by $\alpha$-factor and released into $0.5 \mathrm{M} \mathrm{KCl} \mathrm{YEPD.} \mathrm{Cells} \mathrm{were} \mathrm{collected} \mathrm{at} \mathrm{10-min} \mathrm{intervals} \mathrm{for} 90$ min at $24^{\circ} \mathrm{C}$. Whi5 was analyzed by western blotting as $\mathbf{d}$. $\mathbf{f}$ A cartoon describes the inhibitory pathway of Cip1 to Cdk1-Cln3 complex. The interaction between Cip1 and Cdk1-Cln3 complex inhibits the kinase activity of the Cdk1-Cln3 complex. The inhibition blocks Whi5 phosphorylation, which prevents SBF to transcribe G1/S genes.

g Co-immunoprecipitation between Cip1 and Cln3, Cln3-13A, Cln3-14A, Cln3-24A and Cln3-27A. Immunoprecipitation was conducted using an anti-Myc antibody to pull-down GAL1 promoter-driven Cip1. The band intensities displayed below depict quantification of the relative amount of Cln3 proteins that co-immunoprecipitate with Cip1 


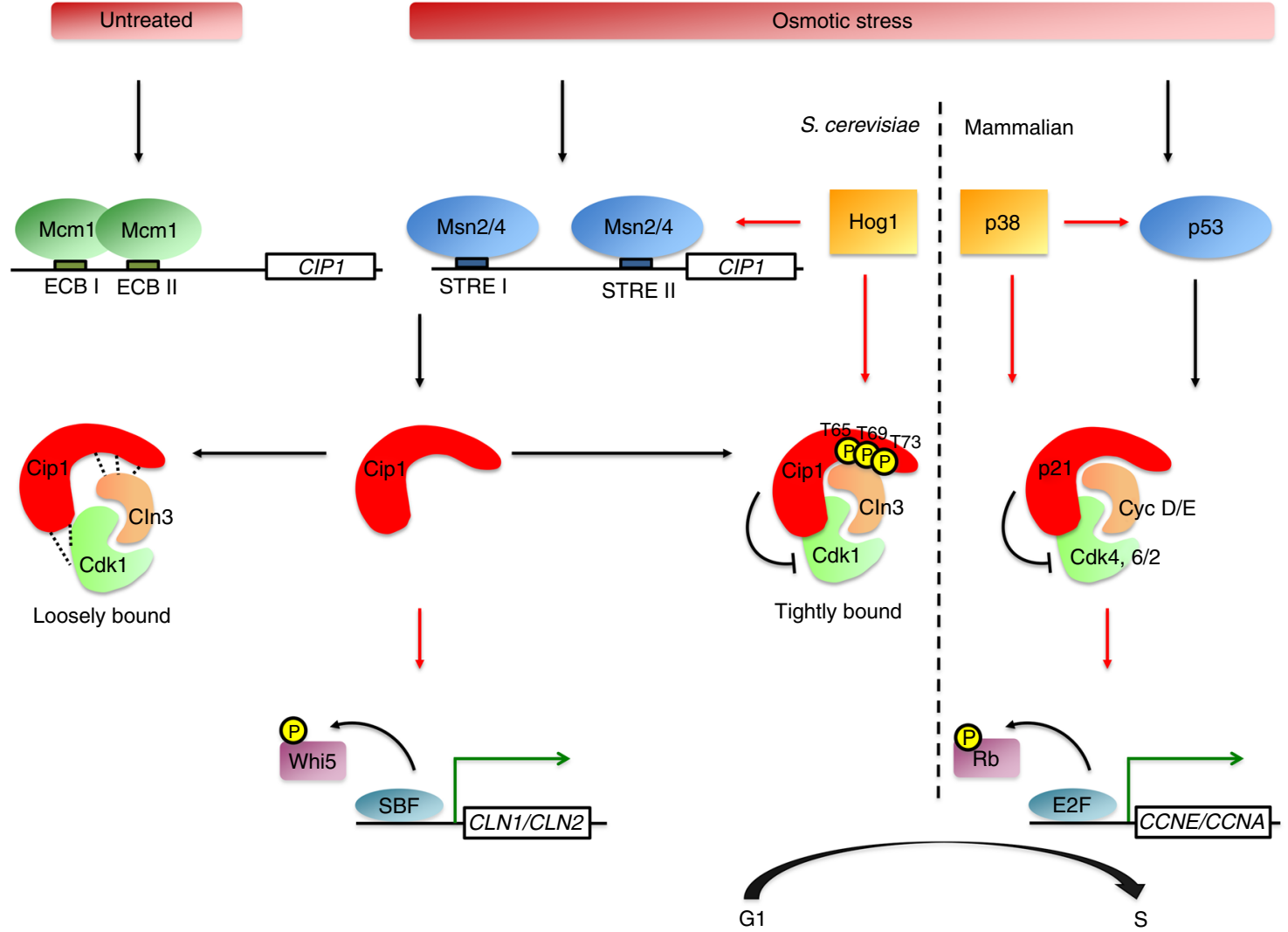

Fig. 8 The Cip1-involved regulatory pathways of G1/S transition controlled by p38/Hog1 SAPKs in mammal/yeast upon osmotic stress. At M/G1 transition, Mcm1 binds at ECB element on CIP1 promoter and promotes the transcriptional expression of CIP1. Upon osmotic stress, active p38 and Hog1 SAPKs phosphorylate p53 and Msn2/4, respectively. In budding yeast, expression of Cip1 is induced by Msn2/4 and expressed Cip1 is phosphorylated at T65, T69, and T73 by Hog1. The phosphorylation of Cip1 strengthens the binding affinity with the Cdk1-Cln3 complex. The interaction between Cip1 and Cdk1-Cln3 complex inhibits the Cdk1-Cln3 kinase activity and prevents Cdk1-Cln3-complex-dependent Whi5 phosphorylation. Non-phosphorylated Whi5 binds to SBF, blocks G1/S genes transcription, and delays G1/S transition. In mammalian cells, the CDK inhibitor p21 is induced by activated p53 which leads to $\mathrm{G} 1$ arrest through inhibition of Cdk4, 6/2-cyclin D/E complexes

Based on the structures and CDK targets, CKIs are divided into two families. The canonical Kip family CKIs bind both cyclin and CDK to block the kinase activity. In contrast, the INK4 family proteins specifically bind CDK monomers, which twists the CDK to disrupt cyclin binding and kinase activity. Overexpressed G1 cyclins can partially overcome the Cip1 block of these cells in G1. On the other hand, Cip1 displays greater association with free form $\mathrm{Cln} 3$. This binding on $\mathrm{Cln} 3$ may impair Cdk1 to form a complex with Cln3. Hence, Cip1is not considered as a canonical CKI.

In a normal cell cycle, Cip1, like Sic1, is expressed at the end of mitosis and persists throughout the G1 phase. However, unlike SIC1 whose expression is not altered under stresses; CIP1 is induced immediately by stresses, including sorbitol-induced hyperosmotic stress, carbon source starvation, oxidative stress and MMS-triggered damage $\mathrm{e}^{51-54}$. Sic1 was previously reported to cause G1 arrest under hyperosmotic stress ${ }^{45}$. Surprisingly, the budding index was additively higher in sic1 cip1 double mutants and loss of CIP1 in sic1 cells further accelerates cell cycle progression after osmotic shock. Thus, Sic1 and Cip1 are functionally redundant CKIs to prevent cells from premature DNA replication when cells encounter environmental challenges. Upon the appearance of environmental stimuli, cells need a swift response to go through the crisis. Deletion of CIP1 further aggravates the stress-sensitive phenotype in sic1 cells. We believe that CIP1 is a stress response gene and its expression slows down G1/S transition to help cells overcome the challenges. Moreover,
Cln overexpression only partially suppresses the Cip1 overexpression effects, suggesting a possibility that Cip1 may also be a $\mathrm{Cdk}-\mathrm{Clb}$ inhibitor to prevent $\mathrm{S}$ phase entry under genotoxic conditions.

In mammalian cells, general stresses activate the SAPK p $38^{55}$. p38 phosphorylates downstream targets which include CKIs p21, p27, and p57 to delay cell cycle progression ${ }^{56-58}$. A recent study revealed that osmotic stress causes a strong G1 delay ${ }^{56,59}$. Inactivation of p38 abolishes osmostress-induced cell-cycle delay and results in decreased cell viability ${ }^{56,59}$. The tumor suppressor protein p53 and its downstream target p21 are the main factors to repress cell cycle under stress ${ }^{60}$. p21 blocks cell cycle and suppresses unwanted cell division ${ }^{61}$. The circuitry controlling this response is conserved from humans to yeast, but no stressinduced negative regulators has been found in yeast before. In yeast, G1 progression upon osmotic stress is contributed by the Hog1 SAPK to phosphorylate Sicl which inhibits Cdk1-Clb activation and promotes Clbs degradation ${ }^{45}, 62$, but the expression of Sic1 is not regulated by stress. Interestingly, Cip1 is also a substrate of Hog1 and it is stress-induced to slow down cell cycle. Furthermore, Cip1 contains a conserved match to the CDK-binding motif found in human $\mathrm{p} 21^{36}$. The function, the SAPK-mediated regulation, and the stress-induced character of Cip1 are partly analogous to those of mammalian p21 in preventing cell cycle entry ${ }^{56-58}$. Moreover, yeast Msn2/Msn4 may act as mammalian p53 in induction of CKI expression to inhibit cell cycle progression under several stresses (Fig. 8). These 
observations extend the parallels between yeast and metazoans at the repression of the cell cycle, and raise the intriguing possibility that the yeast Cip1 may be the primordial one, and the mammalian p21 may be a relative latecomer to the system for stress response.

\section{Methods}

Yeast strains and plasmids. All yeast strains used in this study were derived from

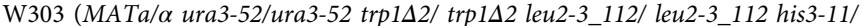

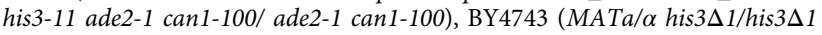

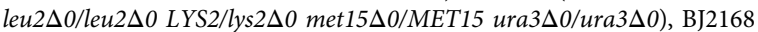
(MATa prcl-407 prb1-1122 pep4-3 leu2 trp1 ura 3-52), CHY125 (MATa ade2-1 ade3::hisG ura3-1 his3-12,15 trp1-1 leu2-3112 can1-100) and YM4271 (MATaura3-

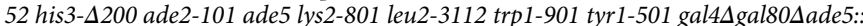
hisG). The deletion strains barl, farl, and sicl are from deletion library (Invitrogen). Mutation strain sic1::LEU2, cip1::KanMX4, and sic1::LEU2cip1::KanMX4 used in budding index assay were generated by introducing mutation cassette into the isogenic BY4742 background. Double mutation strain msn2::KanMX4 msn4:: KanMX4 in BY4742 was selected by tetrad dissection. The tagged strains were created by integration of the $\mathrm{Myc}_{13}$ tag in-frame downstream of specific genes in the genome of BY4741 or W303a $c d c 28$ as-1. The pYESL plasmid contains the pGAL1-CIP1 plasmid, in which the URA3 of pYES2 plasmid (Invitrogen) was destroyed by the LEU2 marker, bearing full-length CIP1 with C-terminal $\mathrm{MYC}_{13}$ tag. The N-terminal GST-tagged Cip1 and p21were expressed from pEGKT plasmid which contains the GAL1 promoter. The pGAL1-CLN1,-CLN2, and -CLN3 plasmids are C-terminal tagged with $\mathrm{HA}_{3}$ in the pYES2 plasmid. For the reporter assays, the $500 \mathrm{bp}$ upstream of the translation start site of CIP1 was PCR-amplified and cloned into placZi (Clontech). Putative ECB elements or STRE sequences in the $\mathrm{pP}_{C I P}$-lacZi reporter plasmid were mutated by site-directed mutagenesis (Invitrogen). Each reporter was introduced into the YM4271 strain as manufacture described (Clontech). All yeast strains, constructs, and primer sequences used in this study are mentioned in Supplementary Tables 1-3, respectively.

RNA preparation and Northern blotting. For total RNA extraction, $5 \mathrm{OD}_{600}$ cells were lysed in $1 \mathrm{ml}$ TRIzol solution (Invitrogen) with glass beads and vigorously vortexed for $5 \mathrm{~min}$ at room temperature. The following manipulations were conducted as described by the manufacturer. For Northern analysis, $5 \mu \mathrm{g}$ of total RNA was separated on a $1.2 \%$ agarose gel containing $2.1 \%$ formaldehyde in MOPS/ formaldehyde buffer. RNA was transferred to a nylon membrane (Perkin-Elmer) and hybridized with $\left[\alpha^{32} \mathrm{P}\right]$ dCTP-labeled probes for CIP1, CLN2, CLN3, CTT1, RNR1, CIT2, and ACT1. Results were analyzed with PhosphorImager and quantitated with ImageJ software. All uncropped Northern blots can be found in Supplementary Fig. 11.

Cell growth media and condition for stress response. The yeast media used were rich medium (YEP, $1 \%$ yeast extract, $2 \%$ peptone) or SC medium containing $2 \%$ glucose, $2 \%$ raffinose, or $2 \%$ galactose as indicated. For Northern blot analysis of cells treated under stress, the overnight culture was grown to early log phase in YEPD at $23^{\circ} \mathrm{C}$. Five $\mathrm{OD}_{600}$ cells were collected at $23^{\circ} \mathrm{C}$ for each indicated time points after exposing to stresses. For carbon source starvation, the glucose concentration was changed from 2 to $0.05 \%$. Osmotic stress was induced by $0.5 \mathrm{M}$ $\mathrm{KCl}$. Heat shock was performed by changing temperature from 23 to $37^{\circ} \mathrm{C}$. The untreated control of rapamycin was supplied with the same volume of dimethyl sulfoxide.

EMSA. The pGEX4T-1-msn2(401-704) plasmid was kindly provided by Dr Ji-Sook Hahn. The GST-Msn2(401-704) protein containing the DNA binding domain of Msn2 was expressed in BL21(DE3) and purified ${ }^{63}$. EMSA was performed as previously described ${ }^{64}$. Briefly, $100 \mathrm{ng}$ recombinant proteins were used. ${ }^{32} \mathrm{P}$-end-labeled double stranded synthetic oligonucleotides containing the STRE I or STRE II were added. The mutant stre I and stre II oligonucleotides were used as negative controls. Following electrophoresis on a $4 \%$ polyacrylamide nondenaturing gel, the gel was dried and subjected to autoradiography.

RNA purification and quantitative reverse transcription PCR. RNA was extracted using TRIzol reagent (Invitrogen). cDNA was synthesized using Thermo Scientific Maxima First Strand cDNA Synthesis (Thermo). Quantitative reverse transcription PCR(qRT-PCR) was performed on a Biorad CFX Connect Real-Time PCR Detection System. All primer sequences for PCR are listed in Supplementary Table 3.

ChIP analysis. ChIP analysis was performed as described ${ }^{65}$. In brief, cells were grown as indicated conditions. After crosslinking in $1 \%$ formaldehyde, cells were lysed and sonicated. Immunoprecipitations were carried out with an anti-Mcm1 antibody (kindly provided by George Sprague) and anti-Msn2 antibody (Santa Cruz, y-300) against Mcm1 and Msn2, respectively. Both an aliquot of sonicated cleared extract (input) and the immunoprecipitated materials were de-crosslinked in Tris/EDTA buffer (TE) plus $1 \%$ SDS for at least $8 \mathrm{~h}$ at $65^{\circ} \mathrm{C}$. Quantification of immunoprecipitated DNA was obtained by qRT-PCR using SYBR Green detection (Kappa) on an Applied Biosystems HT7500 machine and software. After normalized to the background control (promoter of $U 2$ or TEL), the results were expressed as relative fold enrichment binding level on CIP1 promoter. Primers used in this study are listed in the Supplementary Table 3. The data are presented as the mean of at least three synchronies plus or minus s.d.

$\boldsymbol{\alpha}$-Factor/nocodazole trap assay. The $\alpha$-factor/nocodazole trap assay was performed as described ${ }^{66}$. Briefly, after cells were synchronized at G1 by $\alpha$-factor, WT and cipl cells were released in $0.5 \mathrm{M} \mathrm{KCl} \mathrm{YEPD} \mathrm{medium} \mathrm{at} 24^{\circ} \mathrm{C}$. At $15 \mathrm{~min}$ intervals, cells were collected, washed once by YEPD, resuspended in $0.5 \mathrm{ml}$ fresh YEPD combined with $0.5 \mathrm{ml}$ trapping medium $\left(10 \mu \mathrm{M} \alpha\right.$-factor, $30 \mu \mathrm{g} \mathrm{ml}^{-1}$ nocodazole), and incubated at $24^{\circ} \mathrm{C}$ for $90 \mathrm{~min}$. Samples were fixed in $70 \%$ ethanol, sonicated, and examined by phase microscope to count more than 300 cells showing shmoos (G1 cells) or buds (post-G1 cells). The experiment was repeated three times, and representative results are presented.

Budding index under osmotic stress. Wild-type, sic1, cip1, cip1-3TA, or sic1 cip1 strains were grown to exponential phase in YEPD. After treated with $0.5 \mathrm{M} \mathrm{KCl}$, cells were fixed in $70 \%$ ethanol and sonicated. The budding index is defined as the percentage of the whole population carrying a bud, and a total of 400 cells were counted under a DIC microscopy.

Isolation of newborn cells by elutriation. WT, cip1, sic1, and cip1 sic1 strains were pre-grown in YEPD at $30^{\circ} \mathrm{C}$, diluted in YEP-raffinose and grown to $\mathrm{OD}_{660}=2$ before elutriation. Elutriated cells were analyzed at the microscope, and newborn cells were pooled to reach $\mathrm{OD}_{660}=0.6$ and recovered for $90-120 \mathrm{~min}$ in YEPD at $25^{\circ} \mathrm{C}$. At time 0 , cultures were split into halves for $0.5 \mathrm{M} \mathrm{KCl}$ treatment. Cell cycle progression was analyzed by FACS analysis.

Co-immunoprecipitation. The co-immunoprecipitation assay was performed using yeast protease-deficient BJ2168 strains overexpressing Cip1 and cyclin in two different cells. Cells were grown to exponential phase in SC medium with $2 \%$ raffinose, and GAL1 promoter-driven protein was induced by $2 \%$ galactose at $30^{\circ} \mathrm{C} .0 .5 \mathrm{M} \mathrm{KCl}$ was added for $10 \mathrm{~min}$ at $30^{\circ} \mathrm{C}$ to induce phosphorylation of Cip1 Cell pellets were lysed by lysis buffer $(100 \mathrm{mM} \mathrm{NaCl}, 0.1 \%$ Triton X-100, $50 \mathrm{mM}$ Tris- $\mathrm{HCl} \mathrm{pH}$ 7.5). The solubilized fractions containing cyclin or Cipl were mixed and incubated with the antibody-coupled Sepharose beads for $1 \mathrm{~h}$. The binding proteins were separated and detected by western blotting.

MS analysis. To identify the phosphorylation sites of Cip1, GST-Cip1was overexpressed from pEGKT-CIP1 in $0.5 \mathrm{M} \mathrm{KCl}$ treated yeast, isolated by pull-down and SDS-polyacrylamide gel electrophoresis (SDS-PAGE) followed by the in-gel enzyme digestion. The tryptic peptides of Ciplwere analyzed by nanoscale liquid chromatography coupled to tandem mass spectrometry (nano LC-MS/MS) instrument (LTQ-FT, Thermo Fisher Scientific). The MS/MS spectra data were converted as mgf format from experiment RAW file by MM File Conversion Tools $^{67}$ (http://www.massmatrix.net) and then analyzed by MassMatrix ${ }^{68}$ for MS/MS ion search. The search parameters in MassMatrix including the error tolerance of precursor ions and the MS/MS fragment ions in spectra were 10 p.p.m. and $0.6 \mathrm{Da}$. The enzyme for digestion was assigned to be trypsin with the miss cleavage number two. The variable post-translational modifications in search parameters were assigned to include the oxidation of methionine, carbamidomethylation of cysteine, and the phosphorylation of serine/threonine/tyrosine. The phosphorylation sites of Cip1 identified by MS were T65, T69, and T73 (Supplementary Fig. 5c).

Phosphor-specific antibody preparation. Phosphopeptides were conjugated with KLH protein and mixed with Freund's Incomplete Adjuvant (Thermo, Cat.77145) as antigen. New Zealand rabbit was boosted by subcutaneous injected with $0.5 \mathrm{mg}$ per $2 \mathrm{ml}$ antigen every 2 weeks until a sufficient titer. Three days before the final immunization, the rabbit was injected with $0.5 \mathrm{mg}$ antigen without adjuvant into a marginal ear vein. The rabbit was then sacrificed, and blood was collected by cardiac puncture. The blood was incubated at $37^{\circ} \mathrm{C}$ for $1 \mathrm{~h}$ to form clots and incubated at $4{ }^{\circ} \mathrm{C}$ overnight. To separate serum and blood clots, blood was centrifuged at $1,000 \times g$ for $30 \mathrm{~min}$ at $4^{\circ} \mathrm{C}$. The supernatant was collected and stored at $-80^{\circ} \mathrm{C}$.

To improve the titer and specificity of the phosphor-specific antibody, phosphor- or non-phosphopeptides were conjugated with NHS-activated agarose (Thermo, Cat. 26196). After being purified and concentrated by phosphopeptides column and non-phosphopeptides column, the phosphor-specific antibody was stored in $50 \%$ glycerol, $0.05 \%$ sodium azide at $-80^{\circ} \mathrm{C}$.

Dot blot analysis. A quantity of 50, 5, and $0.5 \mathrm{ng}$ of the phosphorylated or unphosphorylated peptides were spotted on nitrocellulose membranes. Dot blot analysis was conducted by standard protocol using phosphor-specific antibodies. 
Western blot analysis. Whole proteins were extracted and resolved by SDS-PAGE, and transferred to a polyvinylidene difluoride membrane. The membrane was blocked in 5\% nonfat milk at room temperature for $1 \mathrm{~h}$, followed by incubation with primary antibody at $4{ }^{\circ} \mathrm{C}$ overnight. Primary antibodies were used to detect HA (Roche, 12CA5, 1:1,000), Myc (Roche, 9E10, 1:1,000), $\beta$-actin (GeneTex, GTX109639, 1:1,000), Cip1 pT65 (1:500), and Cip1 pT73 (1:500). Signals were developed using Luminata ${ }^{\mathrm{TM}}$ Crescendo Western HRP Substrate (Millipore). The image was quantified by ImageJ software. All uncropped western blots can be found in Supplementary Fig. 11.

In vitro kinase assay. GST fusion proteins encoding for Cip1 wild-type, Cip1-3TA, Sic1, Hog1, and Pbs2 ${ }^{\mathrm{EE}}$ were expressed in BL21(DE3) for $5 \mathrm{~h}$ at $16^{\circ} \mathrm{C}$. Cells were lysed by sonication in STET buffer $(10 \mathrm{mM}$ Tris pH $8.0,100 \mathrm{mM} \mathrm{NaCl}$, $1 \mathrm{mM}$ EDTA pH 8.0, 5\% Triton X-100, $2 \mathrm{mM}$ dithiothreitol (DTT), $1 \mathrm{mM}$ phenylmethanesulfonyl fluoride (PMSF), $1 \mathrm{mM}$ benzamidine, $2 \mathrm{mg} \mathrm{ml}^{-1}$ leupeptin, and $2 \mathrm{mg} \mathrm{ml}^{-1}$ of pepstatin). Clear lysates were incubated with glutathioneSepharose beads (GE Healthcare) and GST-tagged proteins were eluted in Elution buffer ( $50 \mathrm{mM}$ Tris $\mathrm{pH} 9.5,2 \mathrm{mM}$ DTT, $10 \mathrm{mM}$ reduced glutathione). One microgram of $\mathrm{Hog} 1$ was activated with $0.5 \mu \mathrm{g}$ of $\mathrm{Pbs} 2^{\mathrm{EE}}$ in the presence of kinase buffer (50 mM Tris- $\mathrm{HCl} \mathrm{pH} \mathrm{7.5,} 10 \mathrm{mM} \mathrm{MgCl}_{2}, 2 \mathrm{mM} \mathrm{DTT}$ ) and $100 \mu \mathrm{M}$ ATP. After $30 \mathrm{~min}$ at $30^{\circ} \mathrm{C}, 2 \mu \mathrm{g}$ of Cipl wild-type, Cip1-3TA or Sicl was added to the $\mathrm{Hog} 1 / \mathrm{Pbs} 2{ }^{\mathrm{EE}}$ mixture together with ${ }^{32} \mathrm{P}$-ATP $(0.1 \mathrm{mCi}$ per $\mathrm{ml})$ and incubated for $30 \mathrm{~min}$ at $30^{\circ} \mathrm{C}$. Hog 1 inhibitor SB203580 (Calbiochem) was added to the relevant samples to a final concentration of $10 \mu \mathrm{M}$ prior to the addition of the substrates to the Hog1/Pbs $2^{\mathrm{EE}}$ mixture. The reaction was terminated by the addition of Laemmli buffer and subsequent boiling. Proteins were resolved in 8\% SDS-PAGE. Gels were stained with Coomassie blue, dried, and analyzed by autoradiography.

Cdk1 in vitro kinase assay was performed as described ${ }^{20,69}$. Briefly, Cln1/Cdk1, $\mathrm{Cln} 2 / \mathrm{Cdk} 1$, and $\mathrm{Cln} 3 / \mathrm{Cdk} 1$ were immunoprecipitated from lysates prepared from BJ2168 bearing pGAL1-CLN1-3HA, pGAL1-CLN2-3HA, or pGAL1-CLN3-3HA, respectively. Recombinant GST and GST-Cip1 proteins were incubated with $\mathrm{Cdk} 1 /$ cyclin immunoprecipitates for $30 \mathrm{~min}$ at $30^{\circ} \mathrm{C}$, and histone $\mathrm{H} 1$ (Sigma, $\mathrm{H} 4524$ ) was used as a kinase substrate. Labeled proteins were separated by $12 \%$ SDS-PAGE, and ${ }^{32} \mathrm{P}$-labeled histone $\mathrm{H} 1$ was visualized by autoradiography and quantitated with a phosphoimager (Molecular Dynamics).

Fluorescence microscopy. Cip1-GFP is driven by CIP1 promoter from a pYEPFAT7 plasmid. Cells overexpressing Cip1-GFP were grown to exponential phase in SC-Ura minus medium. Cip1-GFP was induced in SC-Leu minus medium at $30^{\circ} \mathrm{C}$ for $3 \mathrm{~h} .0 .5 \mathrm{M} \mathrm{KCl}$ was added and incubated at $30^{\circ} \mathrm{C}$ for $5 \mathrm{~min}$. Samples were centrifuged, fixed with $70 \%$ ice-cold ethanol, and washed twice with PBS. DAPI (4',6-diamidino-2-phenylindole) was added to mark the nucleus. The cells were visualized on a Zeiss Imager.M2 fluorescence microscope.

Data availability. The data that supports the findings of this study are available within the article and its supplementary information files or from the corresponding author upon reasonable request.

Received: 13 May 2016 Accepted: 1 June 2017

Published online: 04 July 2017

\section{References}

1. Alexander, M. R. et al. Regulation of cell cycle progression by Swe1p and Hoglp following hypertonic stress. Mol. Biol. Cell 12, 53-62 (2001).

2. Brauer, M. J. et al. Coordination of growth rate, cell cycle, stress response, and metabolic activity in yeast. Mol. Biol. Cell 19, 352-367 (2008).

3. Li, X. \& Cai, M. Recovery of the yeast cell cycle from heat shock-induced G(1) arrest involves a positive regulation of $\mathrm{G}(1)$ cyclin expression by the $\mathrm{S}$ phase cyclin Clb5. J. Biol. Chem. 274, 24220-24231 (1999).

4. Forsburg, S. L. \& Nurse, P. Cell cycle regulation in the yeasts Saccharomyces cerevisiae and Schizosaccharomyces pombe. Annu. Rev. Cell. Biol. 7, 227-256 (1991).

5. Newcomb, L. L., Diderich, J. A., Slattery, M. G. \& Heideman, W. Glucose regulation of Saccharomyces cerevisiae cell cycle genes. Eukaryot. Cell 2, 143-149 (2003).

6. Braun, E. L., Fuge, E. K., Padilla, P. A. \& Werner-Washburne, M. A stationaryphase gene in Saccharomyces cerevisiae is a member of a novel, highly conserved gene family. J. Bacteriol. 178, 6865-6872 (1996).

7. Belli, G., Gari, E., Aldea, M. \& Herrero, E. Osmotic stress causes a G1 cell cycle delay and downregulation of $\mathrm{Cln} 3 / \mathrm{Cdc} 28$ activity in Saccharomyces cerevisiae. Mol. Microbiol. 39, 1022-1035 (2001).

8. de Nadal, E., Ammerer, G. \& Posas, F. Controlling gene expression in response to stress. Nat. Rev. Genet. 12, 833-845 (2011).

9. Shackelford, R. E., Kaufmann, W. K. \& Paules, R. S. Oxidative stress and cell cycle checkpoint function. Free Radic. Biol. Med. 28, 1387-1404 (2000).
10. Cross, F. R. Starting the cell cycle: what's the point? Curr. Opin. Cell Biol. 7, 790-797 (1995).

11. Wittenberg, C., Sugimoto, K. \& Reed, S. I. G1-specific cyclins of S. cerevisiae: cell cycle periodicity, regulation by mating pheromone, and association with the p34CDC28 protein kinase. Cell 62, 225-237 (1990).

12. Tyers, M., Tokiwa, G., Nash, R. \& Futcher, B. The Cln3-Cdc28 kinase complex of $S$. cerevisiae is regulated by proteolysis and phosphorylation. EMBO. J. 11, 1773-1784 (1992).

13. Dirick, L., Bohm, T. \& Nasmyth, K. Roles and regulation of Cln-Cdc28 kinases at the start of the cell cycle of Saccharomyces cerevisiae. EMBO. J. 14, 4803-4813 (1995).

14. Breeden, L. Start-specific transcription in yeast. Curr. Top. Microbiol. Immunol. 208, 95-127 (1996).

15. Costanzo, M. et al. CDK activity antagonizes Whi5, an inhibitor of G1/S transcription in yeast. Cell 117, 899-913 (2004).

16. de Bruin, R. A., McDonald, W. H., Kalashnikova, T. I., Yates, J. 3rd \& Wittenberg, C. Cln3 activates G1-specific transcription via phosphorylation of the SBF bound repressor Whi5. Cell 117, 887-898 (2004).

17. Gonzalez-Novo, A. et al. Hog1 targets Whi5 and Msa1 transcription factors to downregulate cyclin expression upon stress. Mol. Cell Biol. 35, 1606-1618 (2015).

18. Chang, F. \& Herskowitz, I. Identification of a gene necessary for cell cycle arrest by a negative growth factor of yeast: FAR1 is an inhibitor of a G1 cyclin, CLN2. Cell 63, 999-1011 (1990)

19. McKinney, J. D., Chang, F., Heintz, N. \& Cross, F. R. Negative regulation of FAR1 at the Start of the yeast cell cycle. Genes Dev. 7, 833-843 (1993).

20. Peter, M. \& Herskowitz, I. Direct inhibition of the yeast cyclin-dependent kinase Cdc28-Cln by Far1. Science 265, 1228-1231 (1994).

21. Schwob, E., Bohm, T., Mendenhall, M. D. \& Nasmyth, K. The B-type cyclin kinase inhibitor p40SIC1 controls the G1 to S transition in S. cerevisiae. Cell 79, 233-244 (1994).

22. Verma, R., Feldman, R. M. \& Deshaies, R. J. SIC1 is ubiquitinated in vitro by a pathway that requires $\mathrm{CDC} 4, \mathrm{CDC} 34$, and cyclin/CDK activities. Mol. Biol. Cell 8, 1427-1437 (1997).

23. Lengronne, A. \& Schwob, E. The yeast CDK inhibitor Sicl prevents genomic instability by promoting replication origin licensing in late G(1). Mol. Cell $\mathbf{9}$, 1067-1078 (2002).

24. Treisman, R. \& Ammerer, G. The SRF and MCM1 transcription factors. Curr. Opin. Genet. Dev. 2, 221-226 (1992).

25. Riechmann, J. L. \& Meyerowitz, E. M. MADS domain proteins in plant development. Biol. Chem. 378, 1079-1101 (1997).

26. Shore, P. \& Sharrocks, A. D. The MADS-box family of transcription factors. Eur. J. Biochem. 229, 1-13 (1995).

27. Kuo, M. H. \& Grayhack, E. A library of yeast genomic MCM1 binding sites contains genes involved in cell cycle control, cell wall and membrane structure, and metabolism. Mol. Cell. Biol. 14, 348-359 (1994).

28. Lydall, D., Ammerer, G. \& Nasmyth, K. A new role for MCM1 in yeast: cell cycle regulation of SW15 transcription. Genes Dev. 5, 2405-2419 (1991).

29. McInerny, C. J., Partridge, J. F., Mikesell, G. E., Creemer, D. P. \& Breeden, L. L. A novel Mcm1-dependent element in the SWI4, CLN3, CDC6, and CDC47 promoters activates M/G1-specific transcription. Genes Dev. 11, 1277-1288 (1997).

30. MacKay, V. L., Mai, B., Waters, L. \& Breeden, L. L. Early cell cycle box-mediated transcription of CLN3 and SWI4 contributes to the proper timing of the G(1)-to-S transition in budding yeast. Mol. Cell Biol. 21, 4140-4148 (2001).

31. Estruch, F. \& Carlson, M. Two homologous zinc finger genes identified by multicopy suppression in a SNF1 protein kinase mutant of Saccharomyces cerevisiae. Mol. Cell Biol. 13, 3872-3881 (1993).

32. Martinez-Pastor, M. T. et al. The Saccharomyces cerevisiae zinc finger proteins Msn2p and Msn4p are required for transcriptional induction through the stress response element (STRE). EMBO. J. 15, 2227-2235 (1996).

33. Schmitt, A. P. \& McEntee, K. Msn2p, a zinc finger DNA-binding protein, is the transcriptional activator of the multistress response in Saccharomyces cerevisiae. Proc. Natl Acad. Sci. USA 93, 5777-5782 (1996).

34. Causton, H. C. et al. Remodeling of yeast genome expression in response to environmental changes. Mol. Biol. Cell 12, 323-337 (2001).

35. Tkach, J. M. et al. Dissecting DNA damage response pathways by analysing protein localization and abundance changes during DNA replication stress. Nat. Cell Biol. 14, 966-976 (2012).

36. Ren, P., Malik, A. \& Zeng, F. Identification of YPL014W (Cip1) as a novel negative regulator of cyclin-dependent kinase in Saccharomyces cerevisiae. Genes Cells 21, 543-552 (2016).

37. Spellman, P. T. et al. Comprehensive identification of cell cycle-regulated genes of the yeast Saccharomyces cerevisiae by microarray hybridization. Mol. Biol. Cell 9, 3273-3297 (1998).

38. Gorner, W. et al. Nuclear localization of the $\mathrm{C} 2 \mathrm{H} 2$ zinc finger protein Msn2p is regulated by stress and protein kinase A activity. Genes Dev. 12, 586-597 (1998) 
39. Santhanam, A., Hartley, A., Duvel, K., Broach, J. R. \& Garrett, S. PP2A phosphatase activity is required for stress and Tor kinase regulation of yeast stress response factor Msn2p. Eukaryot. Cell 3, 1261-1271 (2004).

40. Smith, A., Ward, M. P. \& Garrett, S. Yeast PKA represses Msn2p/Msn4pdependent gene expression to regulate growth, stress response and glycogen accumulation. EMBO J. 17, 3556-3564 (1998).

41. Schuller, C., Brewster, J. L., Alexander, M. R., Gustin, M. C. \& Ruis, H. The HOG pathway controls osmotic regulation of transcription via the stress response element (STRE) of the Saccharomyces cerevisiae CTT1 gene. EMBO J. 13, 4382-4389 (1994).

42. Rep, M., Krantz, M., Thevelein, J. M. \& Hohmann, S. The transcriptional response of Saccharomyces cerevisiae to osmotic shock. Hotlp and Msn2p/ Msn4p are required for the induction of subsets of high osmolarity glycerol pathway-dependent genes. J. Biol. Chem. 275, 8290-8300 (2000).

43. Hohmann, S. Osmotic stress signaling and osmoadaptation in yeasts. Microbiol. Mol. Biol. Rev. 66, 300-372 (2002).

44. Saito, H. \& Posas, F. Response to hyperosmotic stress. Genetics 192, 289-318 (2012).

45. Escote, X., Zapater, M., Clotet, J. \& Posas, F. Hog1 mediates cell-cycle arrest in G1 phase by the dual targeting of Sic1. Nat. Cell Biol. 6, 997-1002 (2004).

46. Bishop, A. C. et al. A chemical switch for inhibitor-sensitive alleles of any protein kinase. Nature. 407, 395-401 (2000).

47. Miller, M. E., Cross, F. R., Groeger, A. L. \& Jameson, K. L. Identification of novel and conserved functional and structural elements of the G1 cyclin Cln3 important for interactions with the CDK Cdc28 in Saccharomyces cerevisiae. Yeast. 22, 1021-1036 (2005)

48. Ferrigno, P., Posas, F., Koepp, D., Saito, H. \& Silver, P. A. Regulated nucleo/cytoplasmic exchange of HOG1 MAPK requires the importin beta homologs NMD5 and XPO1. EMBO J. 17, 5606-5614 (1998).

49. de Nadal, E. \& Posas, F. Osmostress-induced gene expression--a model to understand how stress-activated protein kinases (SAPKs) regulate transcription. FEBS J. 282, 3275-3285 (2015).

50. Strickfaden, S. C. et al. A mechanism for cell-cycle regulation of MAP kinase signaling in a yeast differentiation pathway. Cell 128, 519-531 (2007).

51. Berry, D. B. \& Gasch, A. P. Stress-activated genomic expression changes serve a preparative role for impending stress in yeast. Mol. Biol. Cell 19, 4580-4587 (2008).

52. Gasch, A. P. et al. Genomic expression programs in the response of yeast cells to environmental changes. Mol. Biol. Cell 11, 4241-4257 (2000).

53. Benton, M. G., Somasundaram, S., Glasner, J. D. \& Palecek, S. P. Analyzing the dose-dependence of the Saccharomyces cerevisiae global transcriptional response to methyl methanesulfonate and ionizing radiation. BMC Genomics 7, 305 (2006).

54. Bradley, P. H., Brauer, M. J., Rabinowitz, J. D. \& Troyanskaya, O. G. Coordinated concentration changes of transcripts and metabolites in Saccharomyces cerevisiae. PLoS Comput. Biol. 5, e1000270 (2009).

55. Cuadrado, A. \& Nebreda, A. R. Mechanisms and functions of p38 MAPK signalling. Biochem. J. 429, 403-417 (2010).

56. Joaquin, $\mathrm{M}$. et al. The p57 CDKi integrates stress signals into cell-cycle progression to promote cell survival upon stress. EMBO J. 31, 2952-2964 (2012).

57. Kim, G. Y. et al. The stress-activated protein kinases p38 alpha and JNK1 stabilize p21(Cip1) by phosphorylation. J. Biol. Chem. 277, 29792-29802 (2002).

58. Todd, D. E. et al. ERK1/2 and p38 cooperate to induce a p21CIP1-dependent G1 cell cycle arrest. Oncogene 23, 3284-3295 (2004).

59. Gubern, A. et al. The N-Terminal phosphorylation of RB by p38 bypasses its inactivation by CDKs and prevents proliferation in cancer cells. Mol. Cell. 64, 25-36 (2016).

60. Rodriguez, R. \& Meuth, M. Chk1 and p21 cooperate to prevent apoptosis during DNA replication fork stress. Mol. Biol. Cell 17, 402-412 (2006).

61. Gartel, A. L. \& Radhakrishnan, S. K. Lost in transcription: p21 repression, mechanisms, and consequences. Cancer. Res. 65, 3980-3985 (2005).

62. Adrover, M. A. et al. Time-dependent quantitative multicomponent control of the $G(1)-S$ network by the stress-activated protein kinase Hog1 upon osmostress. Sci. Signal. 4, ra63 (2011).
63. Lee, P., Cho, B. R., Joo, H. S. \& Hahn, J. S. Yeast Yak1 kinase, a bridge between PKA and stress-responsive transcription factors, Hsf1 and Msn2/ Msn4. Mol. Microbiol. 70, 882-895 (2008).

64. Wong, C. W. et al. Kruppel-like transcription factor 4 contributes to maintenance of telomerase activity in stem cells. Stem Cells 28, 1510-1517 (2010).

65. Shen, Z. J. et al. PP2A and Aurora differentially modify Cdc13 to promote telomerase release from telomeres at G2/M phase. Nat. Commun. 5, 5312 (2014)

66. Wysocki, R., Javaheri, A., Kristjansdottir, K., Sha, F. \& Kron, S. J. CDK Pho85 targets CDK inhibitor Sicl to relieve yeast G1 checkpoint arrest after DNA damage. Nat. Struct. Mol. Biol. 13, 908-914 (2006).

67. Xu, H. \& Freitas, M. A. MassMatrix: a database search program for rapid characterization of proteins and peptides from tandem mass spectrometry data. Proteomics 9, 1548-1555 (2009).

68. Xu, H., Hsu, P. H., Zhang, L., Tsai, M. D. \& Freitas, M. A. Database search algorithm for identification of intact cross-links in proteins and peptides using tandem mass spectrometry. J. Proteome Res. 9, 3384-3393 (2010).

69. Tseng, S. F., Shen, Z. J., Tsai, H. J., Lin, Y. H. \& Teng, S. C. Rapid Cdc13 turnover and telomere length homeostasis are controlled by Cdk1-mediated phosphorylation of Cdc13. Nucleic Acids Res. 37, 3602-3611 (2009).

\section{Acknowledgements}

We thank Drs Jing-Jer Lin and Tsai-Kun Li for valuable inputs, and Drs George F. Sprague and Ji-Sook Hahn for providing materials. We also thank Mariona Nadal-Ribelles for helping in the protein purification and preparing materials of Hogl in vitro kinase assay. This work was supported by grants from the Ministry of Science and Technology (MoST-105-2311-B-002-015-MY3), the National Taiwan University (NTU-ERP-106R8805A1) to Shu-Chun Teng, the Spanish Ministry of Economy and Competitiveness (BFU2015-64437-P and FEDER), the Catalan Government (2014 SGR 599), and the Fundación Botín, by Banco Santander through its Santander Universities Global Division to F.P. F.P. is recipients of an ICREA Acadèmia (Generalitat de Catalunya).

\section{Author contributions}

Y.-L.C., S.-F.T., Y.-C.H., Z.-J.S., P.-H.H., M.-H.H., C.-W.Y., S.T., B.C., L.S., C.-W.W., H.-T.C., and C.-Y.L. conducted and analyzed the experiments. Y.-L.C., S.-F.T., Y.-C.H. Z.-J.S., and S.-C.T. designed the experiments and interpreted the data. Y.-L.C., Z.-J.S., F.P., and S.-C.T. edited the manuscript. S.-C.T. and F.P. provided conceptual advice. S.-C.T. conceived and supervised this study.

\section{Additional information}

Supplementary Information accompanies this paper at doi:10.1038/s41467-017-00080-y.

Competing interests: The authors declare no competing financial interests.

Reprints and permission information is available online at http://npg.nature.com/ reprintsandpermissions/

Publisher's note: Springer Nature remains neutral with regard to jurisdictional claims in published maps and institutional affiliations.

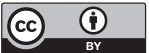

Open Access This article is licensed under a Creative Commons Attribution 4.0 International License, which permits use, sharing, adaptation, distribution and reproduction in any medium or format, as long as you give appropriate credit to the original author(s) and the source, provide a link to the Creative Commons license, and indicate if changes were made. The images or other third party material in this article are included in the article's Creative Commons license, unless indicated otherwise in a credit line to the material. If material is not included in the article's Creative Commons license and your intended use is not permitted by statutory regulation or exceeds the permitted use, you will need to obtain permission directly from the copyright holder. To view a copy of this license, visit http://creativecommons.org/ licenses/by/4.0/.

(c) The Author(s) 2017 